\title{
Gödel's functional interpretation and its use in current mathematics
}

\author{
Ulrich Kohlenbach \\ Fachbereich Mathematik \\ Technische Universität Darmstadt \\ Schlossgartenstraße 7 \\ D-64289 Darmstadt, Germany \\ kohlenbach@mathematik.tu-darmstadt.de \\ http://www.mathematik.tu-darmstadt.de/ ${ }^{\sim}$ kohlenbach/
}

\section{Introduction: general remarks on proof interpretations}

This paper discusses applied aspects of Gödel's functional ('Dialectica') interpretation which originally was designed for foundational purposes. The reorientation of proof theory towards applications to concrete proofs in different areas of mathematics which started in the 50's by G. Kreisel's pioneering work on the 'unwinding of proofs' also led to a re-assessment of possible uses of functional interpretations. Since the 90's this resulted in a systematic development of specially designed versions of functional interpretation and their use in numerical analysis, functional analysis, metric fixed point theory and geodesic geometry. Whereas [67] presents a comprehensive survey of the new results that were obtained in these areas in the course of this investigation, this paper focuses on the underlying logical aspects of these developments. We start, however, with a general discussion of so-called proof interpretations (and their role in Gödel's work) of which functional interpretation is a particularly interesting instance and explain the original motivation behind the latter.

Proof interpretations play an important role in Gödel's work and seem to be used systematically first by him.

Let $\mathcal{T}_{1}$ and $\mathcal{T}_{2}$ be theories in languages $\mathcal{L}\left(\mathcal{T}_{1}\right)$ and $\mathcal{L}\left(\mathcal{T}_{2}\right)$. A proof interpretation $I$ of $\mathcal{T}_{1}$ in $\mathcal{T}_{2}$ in our sense consists of the following elements:

1) to each formula $A \in \mathcal{L}\left(\mathcal{T}_{1}\right)$ a new formula $A^{I} \in \mathcal{L}\left(\mathcal{T}_{2}\right)$ is assigned by induction on the logical structure of $A$.

2) The interpretations of the $\mathcal{T}_{1}$-axioms can be verified in $\mathcal{T}_{2}$.

3) The interpretations of the $\mathcal{T}_{1}$-rules are derivable rules of $\mathcal{T}_{2}$. The most important special case is that of the modus ponens rule:

Modus Ponens Problem: $\frac{A^{I}, \quad(A \rightarrow B)^{I}}{B^{I}}$.

4) Often the soundness proof conveys additional information on $A^{I}$, e.g. a closed term of $\mathcal{L}\left(\mathcal{T}_{2}\right)$ realizing $A^{I}$ in cases where $A^{I}$ is an existential sentence.

As a consequence of these features of proof interpretations one obtains

1) Soundness theorem for $I: \mathcal{T}_{1} \vdash A \Rightarrow \mathcal{T}_{2} \vdash A^{I}$. 
2) A proof $p$ of $A$ can be transformed by $I$ (by recursion on $p$ ) into a proof $p^{I}$ of $A^{I}$. In particular, the main overall logical structure of $p$ remains intact and $p^{I}$ usually is not much longer than $p$.

3) If $\mathcal{L}\left(\mathcal{T}_{1}\right)=\mathcal{L}\left(\mathcal{T}_{2}\right), \mathcal{T}_{2} \subseteq \mathcal{T}_{1}$ and $\Gamma$ is a class of sentences $A$ of $\mathcal{L}\left(\mathcal{T}_{1}\right)$ such that

$$
\mathcal{T}_{2} \vdash A^{I} \rightarrow A,
$$

then $\mathcal{T}_{1}$ is conservative over $\mathcal{T}_{2}$ w.r.t. sentences $\Gamma$. In particular, if $\perp \in \Gamma$ then $I$ yields a relative consistency proof of $\mathcal{T}_{1}$ relative to $\mathcal{T}_{2}$.

In Gödel's work, the following examples of proof interpretations can be found:

1) Gödel's 1933 negative translation of classical ('Peano') arithmetic PA into intuitionistic ('Heyting') arithmetic HA ([32]): $A \mapsto A^{\prime}$ is defined by induction on the logical structure of $A$ as follows:

$$
\begin{aligned}
& P^{\prime}: \equiv \neg \neg P \text { for prime formulas } P, \\
& (A \wedge B)^{\prime}: \equiv A^{\prime} \wedge B^{\prime}, \\
& (A \vee B)^{\prime}: \equiv \neg\left(\neg A^{\prime} \wedge \neg B^{\prime}\right), \\
& (A \rightarrow B)^{\prime}: \equiv \neg\left(A^{\prime} \wedge \neg B^{\prime}\right), \\
& (\forall x A)^{\prime}: \equiv \forall x A^{\prime}, \\
& (\exists x A)^{\prime}: \equiv \neg \forall x \neg A^{\prime} .
\end{aligned}
$$

In [32], Gödel proved that

$$
\text { (*) } \mathrm{PA} \vdash A \Rightarrow \mathrm{HA} \vdash A^{\prime} \text {. }
$$

This establishes the consistency of classical arithmetic relative to intuitionistic arithmetic and (using that intuitionistically $(A \rightarrow B)^{\prime} \leftrightarrow\left(A^{\prime} \rightarrow B^{\prime}\right)$ ) the conservativity of the former over the latter for so-called $\exists$-free sentences, i.e. sentences which neither contain $\exists$ nor $\vee$.

The 'characterization theorem' for negative translation is trivial:

$$
\mathrm{PA} \vdash A \leftrightarrow A^{\prime}
$$

and so also the converse of $(*)$ holds.

A similar such translation was found independently by G. Gentzen and, prior to Gödel, for propositional logic V.I. Glivenko [31] had shown that the simple translation $A \mapsto \neg \neg A$ already suffices. Other variants were subsequently developed by S. Kuroda ([86]) and J.L. Krivine (made explicit in [105] and [104]). Negative translations have been extended to a great variety of other systems (see A.S. Troelstra's introductory notes to Gödel's paper in [39]).

2) Gödel's 1933 interpretation of intuitionistic propositional logic into classical modal propositional logic S4 ([33]): Let IPC denote intuitionistic propositional logic and S4 the familiar modal proposition logic defined by C.I. Lewis. Now define for $A \in \mathcal{L}($ IPC $) A^{\square}$ by induction on $A$ :

$$
\begin{aligned}
& P^{\square}: \equiv P \text { for atomic sentences } P, \\
& (A \vee B)^{\square}: \equiv\left(\square A^{\square} \vee \square B^{\square}\right), \\
& (A \wedge B)^{\square}: \equiv\left(A^{\square} \wedge B^{\square}\right), \\
& (A \rightarrow B)^{\square}: \equiv \square A^{\square} \rightarrow \square B^{\square}, \\
& (\neg A)^{\square}: \equiv \neg \square A^{\square} .
\end{aligned}
$$


The following is shown in [33]:

$$
\mathrm{IPC} \vdash A \Rightarrow \mathrm{S} 4 \vdash A^{\square}
$$

The converse implication (conjectured in [33]) was proved in [92]. The resulting equivalence was extended to predicate logic in [96] and [91] independently (taking $(\forall x A)^{\square}: \equiv \forall x A^{\square}$ and $\left.(\exists x A)^{\square}: \equiv \exists x \square A^{\square}\right)$.

These results give a kind of interpretation of intuitionistic logic (as well as intuitionistic arithmetic and analysis to which this interpretation was extended subsequently in various ways) in classical terms as a logic of proof obligations. E.g. intuitionistically, a sentence $A \vee B$ asks for a proof of either $A$ or $B$. Here 'provability of $A$ ' (i.e. $\square A$ ) must not be understood w.r.t. a fixed formal system such as Peano arithmetic PA since the S4-axiom $\square A \rightarrow A$ would - as a consequence of Gödel's 2nd incompleteness theorem - not be valid under such an interpretation. This, however, can be overcome by refining the interpretation in terms of provability into a logic of proofs which was sketched by Gödel in 1938 (see [34], published only in 1995 in [41]) and fully elaborated by S. Artemov in [2].

3) Gödel's functional ('Dialectica') interpretation ([37], see also [36] and [38]) gives an interpretation of HA into a quantifier-free calculus $T$ of Hilbert's ([48]) primitive recursive functionals of finite type. It is based on a formula assignment

$$
A \mapsto \exists \underline{x} \forall \underline{y} A_{D}(\underline{x}, \underline{y}),
$$

where $A_{D}$ is quantifier-free and $\underline{x}, \underline{y}$ are tuples of functionals of finite type, together with a soundness proof which shows by induction on a given HA-proof of $A$ how to construct closed terms $\underline{t}$ of $T$ such that

$$
T \vdash A_{D}(\underline{t}, \underline{y}) .
$$

Since $0=1$ is preserved by this interpretation, the result provides a consistency proof for HA (and so by the negative translation discussed in 1) above also for PA) relative to $T$. The foundational significance of this reduction rests on the fact that $T$ contains only (a rule of) quantifier-free induction, though stated in an extended language of functionals of finite types. The discussion of this technique and its applications to mathematics in more recent years will be the main issue of the rest of this paper.

A common feature of all three proof interpretations, but also of Gödel's inner model construction for $V=L$ in [35], is the treatment of constructive reasoning from a classical standpoint:

- The negative translation shows that (irrespectively of the totally different philosophical concepts behind classical and intuitionistic mathematics) they can rather easily be related to each other in the sense that intuitionistic arithmetic can be viewed as a refinement of classical arithmetic which contains the latter in its $\exists$-free part but which makes finer distinctions in general, e.g. by distinguishing the classical $\exists$-quantifier ' $\neg \forall \neg$ ' from a constructive one.

- The interpretation of IPC into S4 shows that the refinement provided by intuitionistic logic can be accounted for also in a classical setting provided the latter is extended by a suitable modal operator.

- In the next section we will argue that Gödel's functional interpretation can be viewed as treating the intended meaning of the intuitionistic connectives as spelled out by the so-called Brouwer-Heyting-Kolmogorov interpretation from a classical point of view in which also negatively occurring universal quantifiers count as existential ones and so only purely universal statements carry complete information (compare this with the 'real statements' of Hilbert). To put it in different terms: Gödel uses a classical concept of ' $\exists$-free' within an interpretation of intuitionistic arithmetic. 
- In Gödel's work on $V=L$ the concept of predicative definability (developed in the 'semiintuitionistic' context put forward by H. Weyl, E. Borel, H. Poincaré and others) is used within impredicative classical set theory. In a letter to H. Wang from March 7, 1968 Gödel wrote

'However, as far as, in particular, the continuum hypothesis is concerned, there was a special obstacle which really made it practically impossible for constructivists to discover my consistency proof. It is the fact that the ramified hierarchy, which had been invented expressly for constructivistic purposes, has to be used in an entirely nonconstructive way.' Gödel [110],p.205 (reprinted in: [43], p.404).

This, however, seems to be somewhat too strongly worded: after all Gödel's consistency proof has a constructive interpretation as a relative consistency proof of $\mathrm{GB}+\mathrm{GCH}$ relative to $\mathrm{GB}(=\mathrm{Gödel}-$ Bernays set theory), see also Kreisel [83]. In his 'Lecture on the consistency of the continuums hypothesis' at Brown University (published posthumously in [41], pp. 175-185) he explicitly links his consistency proof to the program (for a 'proof' of the continuums hypothesis) outlined in Hilbert [48]:

'Just recently I have succeeded in giving the proof a new shape which makes it somewhat similar to Hilbert's program presented in his lecture "Über das Unendliche" [48]' Gödel ([41], p.175).

In addition to this it is worth noting that in the letter to Wang cited above Gödel also attributes the fact that neither Skolem nor Herbrand had discovered his completeness theorem to 'that reluctance to use non-finitary concepts and arguments in metamathematics' ([43], pp. 403-404). However, there is evidence (see Goldfarb's introduction to [44], p.12) that Herbrand was aware of the possibility to use an ineffective argument to derive the completeness theorem from his theorem but did not believe that general 'validity' would make sense and focussed on the fact that a particular set of rules (having the subformula property by omitting the syllogism rule) was as strong as the usual rules:

'the theorem in question permits us to show that the system of rules of reasoning can be changed profoundly while still remaining equivalent to the original ones, so that the rule of the syllogism, the basis of Aristotelian logic, is of no use in any mathematical argument' (Herbrand [44], p.276).

\section{Functional Interpretation}

Functional interpretation was developed by Gödel for intuitionistic arithmetic HA but - via his negative translation - also applies to classical arithmetic PA. We start by briefly recalling the wellknown Brouwer-Heyting-Kolmogorov ('BHK') interpretation of intuitionistic logic in terms of 'proofs' which rather than proofs in a specific formal systems should be viewed as 'verifying constructions':

1) A proof of $A \wedge B$ is a pair $\left(p_{A}, p_{B}\right)$, where $p_{A}$ is a proof of $A$ and $p_{B}$ is a proof of $B$.

2) A proof of $A \vee B$ is a pair $(n, p)$, where $n$ is a natural number and $p$ is a proof of $A$ if $n=0$ and a proof of $B$ if $n \neq 0$ respectively.

3) A proof of $A \rightarrow B$ is a construction $p$ that transforms any hypothetical proof $q$ of $A$ into a proof $p(q)$ of $B$.

4) A proof of $\exists x A(x)$ is a pair $(c, p)$, where $c$ is an element (or - more precisely - a term denoting an element) of the underlying domain and $p$ is a proof of $A(c)$.

5) A proof of $\forall x A(x)$ is a construction $p$ that transforms any element $c$ of the underlying domain into a proof $p(c)$ of $A(c)$.

Negation is treated as a defined notion $\neg A: \equiv(A \rightarrow \perp)$ with the stipulation that there is no proof $p$ for $\perp$. 
This informal interpretation has some ambiguities, in particular in the clause for implication. Making this precise results in various so-called realizability interpretations. The most important ones are Kleene's realizability ([54]) and Kreisel's modified realizability ([78, 79]). Let us sketch now the main differences between these two interpretations:

- Kleene's realizers are natural numbers viewed as codes of partial recursive functions. A number $x$ realizes an implication $A \rightarrow B$ (short: $x r(A \rightarrow B)$ ) if

$$
\forall y \in \mathbb{N}(y r A \rightarrow\{x\}(y) \downarrow \wedge\{x\}(y) r B),
$$

i.e.

$$
\forall y \in \mathbb{N}(y r A \rightarrow \exists z \in \mathbb{N}(\{x\}(y) \simeq z \wedge z r B)) .
$$

In particular, a Kleene-realizer does not have to be defined in general on hypothetical realizers for $A$ but only on actual realizers for $A$. Referring to the standard formalization of elementary recursion theory in $\mathrm{HA}$, the interpretation $x r A$ of a formula in the language of HA again is a formula in that language.

- In contrast to the partial (and type-free) Kleene realizability, realizers in the sense of modified realizability are (tuples of) total functionals $\underline{x}$ of finite type (in a suitable domain of functionals) such that (denoting modified realizability by ' $m r$ ')

$$
\underline{x} m r(A \rightarrow B): \equiv \forall \underline{y}(\underline{y} m r \quad A \rightarrow \underline{x}(\underline{y}) m r B)
$$

where for $\underline{x}=x_{1}, \ldots, x_{n}$ the expression $\underline{x}(\underline{y})$ denotes the tuple $x_{1}(\underline{y}), \ldots, x_{n}(\underline{y})$. To make this precise we need to define a finite type extension $\mathrm{HA}^{\omega}$ of HA such that for $\bar{A}$ in the language of HA (and even for $A$ in the extended language of HA ${ }^{\omega}$ ) the interpretation $\underline{a} m r A$ of $A$ is a formula of the language of $\mathrm{HA}^{\omega}$.

Both, the formula $x$ r $A$ as well as the formula $\underline{x} \operatorname{mr} A$ are in general not quantifier-free since in both cases the clause for implication (as well as for the universal quantifier) contains a universal quantifier. However, ' $\underline{x} m r A^{\prime}$ ' is a so-called $\exists$-free formula, i.e. a formula that does not contain $\vee$ or $\exists$ (note that over HA, $A \vee B$ is equivalent to $\exists n[(n=0 \rightarrow A) \wedge(n \neq 0 \rightarrow B)]$ and so is usually counted as an existential quantifier).

Due to the existential quantifier hidden in $\{x\}(y) \downarrow$ the formula ' $x r A$ ' is only 'essentially $\exists$-free', i.e. does not contain $\vee$ and contains $\exists$ only in front of prime formulas.

As mentioned already above, Gödel's functional interpretation $A^{D}$ can be viewed as a form of modified realizability where all classical existential quantifiers (i.e. not only positively occurring $\exists$-quantifiers but also negatively occurring $\forall$-quantifiers) are included among the data $\exists \underline{x}$ that need to be realized leaving only a purely universal formula left, i.e. $A^{D} \equiv \exists \underline{x} \forall \underline{y} A_{D}(\underline{x}, \underline{y})$, where $A_{D}$ is a quantifier-free formula. ${ }^{1}$ This requires a more subtle interpretation of implications: not only is an implication

$$
\exists x A_{0}(x) \rightarrow \exists y B_{0}(y)
$$

$\left(A_{0}, B_{0}\right.$ quantifier-free) interpreted (following $m r$ ) as

$$
\exists f \forall x\left(A_{0}(x) \rightarrow B_{0}(f(x)),\right.
$$

but, in addition, an implication

$$
\forall x A_{0}(x) \rightarrow \forall y B_{0}(y)
$$

\footnotetext{
${ }^{1}$ Some clarification of the relationship between modified realizability and functional interpretation has been achieved in [103] where an infinite family of interpretations in between these two is introduced (see also [93]). However, in our view functional interpretation is rather different from any realizability notion. E.g. realizability notions all have a so-called 'with-truth'-variant due to P. Aczel (see [109]) whereas such a variant of (the Diller-Nahm version [19] of) $D$ is not sound (while a closely related so-called $q$-variant due to Kleene is) as was shown in [52, 53].
} 
needs to be interpreted as

$$
\exists g \forall y\left(A_{0}(g(y)) \rightarrow B_{0}(y)\right) .
$$

In general, the functional interpretation of implications goes as follows: suppose that we have defined already functional interpretations $A^{D} \equiv \exists \underline{x} \forall \underline{y} A_{D}(\underline{x}, \underline{y})$ and $B^{D} \equiv \exists \underline{u} \forall \underline{v} B_{D}(\underline{u}, \underline{v})$. Then the interpretation $(A \rightarrow B)^{D}$ of $A \rightarrow B$ is defined as

$$
(*) \exists \underline{U}, \underline{Y} \forall \underline{x}, \underline{v}\left(A_{D}(\underline{x}, \underline{Y}(\underline{x} \underline{v})) \rightarrow B_{D}(\underline{U}(\underline{x}), \underline{v})\right) .
$$

This is motivated as follows: among all four possibilities of prenexing

$$
\exists \underline{x} \forall \underline{y} A_{D}(\underline{x}, \underline{y}) \rightarrow \exists \underline{u} \forall \underline{v} B_{D}(\underline{u}, \underline{v})
$$

one chooses

$$
\forall \underline{x} \exists \underline{u} \forall \underline{v} \exists \underline{y}\left(A_{D}(\underline{x}, \underline{y}) \rightarrow B_{D}(\underline{u}, \underline{v})\right) .
$$

Now applying the axiom of choice (see below) for functionals of arbitrary type yields $(*$ ). If we had chosen any of the three remaining possibilities for the prenexation, the resulting functional interpretation would fail to have computable realizers already for certain instances of $A \rightarrow A$ (see $[108](3.5 .3))$.

As we see, functionals of higher type show up (both in the course of modified realizability as well as functional interpretation) even for formulas in the language of HA (i.e. formulas which only involve variables of the lowest type) and, in fact, the realizing terms will be so-called primitive recursive functionals in the extended sense of [48] and [37]: the set $\mathbf{T}$ of finite types is generated inductively by the following clauses

$$
\text { (i) } 0 \in \mathbf{T}, \quad(i i) \rho, \tau \in \mathbf{T} \Rightarrow(\rho \rightarrow \tau) \in \mathbf{T} .
$$

0 is the type of natural numbers and $(\rho \rightarrow \tau)$ the type of objects mapping objects of type $\rho$ to objects of type $\tau$. The degree $\operatorname{deg}(\rho)$ of $\rho$ is defined by $\operatorname{deg}(0):=0, \operatorname{deg}(\rho \rightarrow \tau):=\max \{\operatorname{deg}(\rho)+1, \operatorname{deg}(\tau)\}$. Usually outmost parentheses are omitted. The types $0 \rightarrow 0$ and $(0 \rightarrow 0) \rightarrow 0$ usually are denoted by 1 and 2 respectively. The primitive recursive functionals of finite type in the sense of Hilbert [48] and [37] are generated starting from $0^{0}$ (zero), $S^{(0 \rightarrow 0)}$ (successor) and variables of arbitrary types by the following two schemas

- For each term $t^{\tau}[\underline{u}]$ built up out of $0, S$, previously defined primitive recursive functionals and at most the variables $\underline{u}=u_{1}^{\rho_{1}}, \ldots, u_{k}^{\rho_{k}}$ there is a primitive recursive functional $\varphi$ such that

$$
\forall \underline{u}\left(\varphi(\underline{u})={ }_{\tau} t[\underline{u}]\right) .
$$

- For previously defined primitive recursive functionals $\psi^{\tau}$ and $\chi^{\tau \rightarrow(0 \rightarrow \tau)}$ there is a primitive recursive functional $\varphi$ such that

$$
\varphi\left(0^{0}\right)={ }_{\tau} \psi, \quad \forall u^{0}\left(\varphi(S(u))={ }_{\tau} \chi(\varphi(u), u)\right) .
$$

A possible domain to interpret these functionals over is the full set-theoretic type structure $\mathcal{S}^{\omega}=$ $\left\langle S_{\rho}\right\rangle_{\rho \in \mathbf{T}}$, where $S_{0}:=\mathbb{N}$ and $S_{\rho \rightarrow \tau}$ is the set of all set-theoretic functionals $S_{\rho} \rightarrow S_{\tau}$. As observed already by D. Hilbert in [48] the above form of primitive recursion in higher types which allows one to use the whole functional $\varphi(u)$ of type $\tau$ in each step of the recursion to define $\varphi(S(u))$ makes it possible to define more functions $f: \mathbb{N} \rightarrow \mathbb{N}$ than just the ordinary primitive recursive ones, namely e.g. the well-known Ackermann function and - as shown in [37] (see below) - in fact all provably total recursive functions of $\mathrm{PA}$.

It turns out that the same definition of functional interpretation applies to formulas formulated already in an extension HA ${ }^{\omega}$ of HA to all finite types. However, in order to generalize Gödel's soundness theorem for the interpretation from $\mathrm{HA}$ to $\mathrm{HA}^{\omega}$ one has to observe two subtle points: 
1) Since the functional interpretation of the contraction axiom $A \rightarrow A \wedge A$ depends on the existence of decision functionals for the prime formulas involved, one must not include higher type equality relations $=_{\rho}$ as primitive concepts to the language unless one has an intensional interpretation of $=\rho$ in mind for which it is reasonable to assume the existence of effective decision functions.

2) If only $=_{0}$ is taken as a primitive notion (so that the only prime formulas are $s={ }_{0} t$ which are decidable) higher type equality has to be defined either as

(a) extensional equality $s={ }_{\rho} t: \equiv \forall \underline{v}\left(s(\underline{v})={ }_{0} t(\underline{v})\right)$ with new variables $\underline{v}$ so that $s(\underline{v})$ is of type 0 or

(b) in a way that is neutral w.r.t. the issue whether $s={ }_{\rho} t$ is interpreted extensionally or intensionally, namely as Leibniz identity, i.e. indistinguishability in all number contexts

$$
r\left[s / x^{\rho}\right]={ }_{0} r\left[t / x^{\rho}\right]
$$

for all terms $r^{0}$ of type 0 .

Whereas Gödel in [37] apparently had an intensional interpretation of higher type equality in mind, in his earlier treatment of 1941 ([36]) he argued for the option to include only equality for numbers as a primitive notion (see the introductory remarks to [37] by A.S. Troelstra in [41]). We will take this option here and treat higher type equality as extensional equality as this is the most natural interpretation for applications in mathematics. Then, however, in order for the soundness theorem to hold one must not stipulate as an axiom that all functionals respect this extensionality but only a weaker rule version of this (first considered in [102]):

$$
\text { QF-ER : } \frac{A_{0} \rightarrow s={ }_{\rho} t}{A_{0} \rightarrow r\left[s / x^{\rho}\right]={ }_{\tau} r[t / x]},
$$

where $A_{0}$ is quantifier-free. We denote the resulting system (usually called 'WE-HA' ${ }^{\omega}$ ' in the literature, see e.g. [108],[3] or [69] for precise definitions) by $\mathrm{HA}^{\omega}$ since in this paper there is no danger to confuse this with other variants. qf-HA ${ }^{\omega}$ is the quantifier-free fragment of $\mathrm{HA}^{\omega}$, formulated with a substitution rule

$$
\text { SUB : } \frac{A}{A\left[t^{\rho} / x^{\rho}\right]}
$$

and a quantifier-free rule of induction

$$
\text { QF-IR }: \frac{A(0), A(x) \rightarrow A(S(x))}{A(x)}
$$

instead of the schema of full induction, both for all formulas $A$ in this quantifier-free language. Except for our extensional treatment of higher type equality (now formulated as open formulas without quantifiers) this system is called calculus $T$ in [37].

Definition 2.1. 1) The so-called Markov Principle in all finite types is the schema

$$
\mathrm{M}^{\omega}: \neg \neg \underline{\exists} A_{0}(\underline{x}) \rightarrow \exists \underline{x} A_{0}(\underline{x}),
$$

where $A_{0}$ is an arbitrary quantifier-free formula of $\mathrm{HA}^{\omega}$ and $\underline{x}$ is a tuple of variables of arbitrary types $\left(A_{0}(\underline{x})\right.$ may contain further free variables in addition to $\left.\underline{x}\right)$.

2) The independence-of-premise schema $\operatorname{IP}_{\forall}^{\omega}$ for universal premises is the schema

$$
\operatorname{IP}_{\forall}^{\omega}:\left(\forall \underline{x} A_{0}(\underline{x}) \rightarrow \exists y B(y)\right) \rightarrow \exists y\left(\forall \underline{x} A_{0}(\underline{x}) \rightarrow B(y)\right),
$$

where $A_{0}$ is quantifier-free, $y, \underline{x}$ have arbitrary types and $y$ does not occur free in $\forall \underline{x} A_{0}(\underline{x})$. 
3) The axiom of choice schema $\mathrm{AC}$ in all types $\rho, \tau$ is given by

$$
\mathrm{AC}: \forall x^{\rho} \exists y^{\tau} A(x, y) \rightarrow \exists Y^{\rho \rightarrow \tau} \forall x^{\rho} A(x, Y(x)),
$$

where $A$ is an arbitrary formula (not containing $Y$ free).

4) The axiom of quantifier-free choice schema QF-AC in all types is given by

$$
\mathrm{QF}-\mathrm{AC}: \forall \underline{x} \exists \underline{y} A_{0}(\underline{x}, \underline{y}) \rightarrow \exists \underline{Y} \forall \underline{x} A_{0}(\underline{x}, \underline{Y}(\underline{x})),
$$

where $A_{0}$ is a quantifier-free formula (not containing $\underline{Y}$ free) and $\underline{x}, \underline{y}$ are tuples of variables of arbitrary types.

Notation: In the following let $\mathrm{H}^{\omega}:=\mathrm{HA}^{\omega}+\mathrm{AC}+\mathrm{IP}_{\forall}^{\omega}+\mathrm{M}^{\omega}$.

Theorem 2.2 (soundness of functional interpretation [37],[111],[108]). The following rule holds:

$$
\mathrm{H}^{\omega} \vdash A(\underline{a}) \text { implies that } T \vdash A_{D}(\underline{t}(\underline{a}), \underline{y}, \underline{a}),
$$

where $\underline{t}$ is a suitable tuple of closed terms of $\mathrm{HA}^{\omega}$ which can be extracted from a given proof of the assumption and $\underline{a}$ contains all free variables of $A$.

Combined with Gödel's negative translation $A \mapsto A^{\prime}$ which extends from PA to the classical variant $\mathrm{PA}^{\omega}$ of $\mathrm{HA}^{\omega}$ (i.e. $\mathrm{HA}^{\omega}$ with the law-of-excluded-middle schema $A \vee \neg A$ added) it follows that (see e.g. [89] or [69]):

Theorem 2.3. The following rule holds:

$$
\mathrm{PA}^{\omega}+\mathrm{QF}-\mathrm{AC} \vdash A(\underline{a}) \text { implies that } T \vdash\left(A^{\prime}\right)_{D}(\underline{t}(\underline{a}), \underline{y}, \underline{a}),
$$

where $\underline{t}$ is a suitable tuple of closed terms of $\mathrm{HA}^{\omega}$ which can be extracted from a given proof of the assumption.

Remark 2.4. 1) The extraction of the terms $\underline{t}$ in both theorems is carried out by recursion over the given proof. The complexity of this extraction procedure is rather low: the size of the extracted terms is linear in the size of the given proof, the extraction algorithm has a cubic worst-time complexity and the depth of the verifying proof is linear in the depth of the given proof and the maximal size of formulas occurring in that proof (see [47] for this and much more detailed information). The extraction algorithm has been further optimized in the 'Light Functional Interpretation' of [45] and is implemented in [46].

2) In general, $\left(A^{\prime}\right)^{D}$ causes unnecessarily high types. More efficient is e.g. to use Kuroda's negative translation ([86]) instead of Gödel's original interpretation which subsequently was further optimized by Krivine (see [85, 105])). In fact, the combination of Krivine's negative interpretation with functional interpretation yields precisely the so-called Shoenfield variant ([99]) of Gödel's functional interpretation (see [104]).

Corollary 2.5. PA $\vdash 0=1 \Rightarrow T \vdash 0={ }_{0} 1$.

This corollary, which provides a relative consistency proof of PA relative to $T$, is the main objective in [37]. The significance of the proof-theoretic reduction achieved by this rests on the fact that induction for arbitrarily complex formulas is reduced to quantifier-free induction (though formulated in an extended language of primitive recursive functionals of finite type).

A far reaching extension of Gödel's result was obtained in 1962 by C. Spector ([102]) who succeeded to give a functional interpretation of classical analysis $\mathcal{A}^{\omega}$ axiomatized as the extension of $\mathrm{PA}^{\omega}+\mathrm{QF}^{-}$ $\mathrm{AC}$ by the axiom schema of countable choice

$$
\mathrm{AC}^{0, \rho}: \forall x^{0} \exists y^{\rho} A(x, y) \rightarrow \exists f^{0 \rightarrow \rho} \forall x^{0} A(x, f(x)),
$$


where $A$ is an arbitrary formula of $\mathrm{PA}^{\omega}$ (not containing $f$ free). The functional interpretation is carried out in the extension $T+\mathrm{BR}$ of $T$ by a schema BR of so-called bar recursion. In fact, the interpretation also works for the axiom schema of dependent choice DC $([49,89,69])$. This yields a relative consistency result of classical analysis relative to $T+\mathrm{BR}$. Further extensions have been given in e.g. $[29,24,15]$. Moreover, functional interpretation has been adapted to fragments of $\mathrm{PA}^{\omega}$ based on restricted forms of induction and could be used to calibrate the provably recursive function(al)s of such fragments (see $[95,17])$.

\section{From consistency proofs to the unwinding of proofs}

For applications of functional interpretation to mathematics with the aim of extracting new effective data from a given proof, the reduction of full induction to the rule of quantifier-free induction QF-IR is rather irrelevant whereas now the emphasis is on the extraction of terms realizing $A^{D}$ resp. $\left(A^{\prime}\right)^{D}$ for interesting theorems $A$. This 'shift of emphasis' (G. Kreisel) also leads to the following observation stressed by G. Kreisel already in the 50's: whereas in reductive proof theory the provability of universal sentences, namely formalized consistency statements, is a main focus of interest, one may add arbitrary true universal sentences to e.g. PA or PA ${ }^{\omega}$ as axioms without any effect on the extractable programs from proofs. In particular, this means that in this connection proofs of purely universal lemmas do not need to be analyzed at all. Before we can state the main consequences of the (proof of the) soundness theorem of functional interpretation for the extractability of programs we need the following characterization result for $A^{D}$ :

Proposition 3.1 ([111],[108]). For all formulas $A$ of $\mathcal{L}\left(\mathrm{HA}^{\omega}\right)$ one has

$$
\mathrm{H}^{\omega} \vdash A \leftrightarrow A^{D}
$$

Theorem 3.2 (Program extraction by D-interpretation). Let $\mathcal{P}$ be an arbitrary set of purely universal sentences $\forall \underline{a} \underline{\underline{\sigma}} P_{0}(\underline{a})$ ( $P_{0}$ quantifier-free) of $\mathcal{L}\left(\mathrm{HA}^{\omega}\right)$ and $A_{0}\left(x^{\rho}, u^{\delta}\right)$ be quantifier-free formula containing only $x, u$ free and $B\left(x^{\rho}, y^{\tau}\right)$ an arbitrary formula containing only $x, y$ free and $\rho, \delta, \tau$ are arbitrary types. Then the following rule holds:

$$
\left\{\begin{array}{l}
\mathrm{H}^{\omega}+\mathcal{P} \vdash \forall x^{\rho}\left(\forall u^{\delta} A_{0}(x, u) \rightarrow \exists y^{\tau} B(x, y)\right) \\
\text { then one can extract a closed term } t \text { of } \mathrm{HA}^{\omega} \text { s.t. } \\
\mathrm{H}^{\omega}+\mathcal{P} \vdash \forall x^{\rho}\left(\forall u^{\delta} A_{0}(x, u) \rightarrow B(x, t(x))\right) .
\end{array}\right.
$$

In particular, if $\mathcal{S}^{\omega} \models \mathcal{P}$, then the conclusion holds in $\mathcal{S}^{\omega}$, where $\mathcal{S}^{\omega}$ is the full set-theoretic model of $\mathrm{PA}^{\omega}$ (and hence - using $\mathrm{AC}$ on the metalevel - of $\mathrm{H}^{\omega}$ ).

The result also holds for tuples of variables $\underline{x}, \underline{u}, \underline{y}$ where then $t$ is a tuple of closed terms.

Proof (see also [108](3.7.5)): Making use of $\operatorname{IP}_{\forall}^{\omega}$ the assumption yields that

$$
\mathrm{H}^{\omega}+\mathcal{P} \vdash \forall x^{\rho} \exists y^{\tau}\left(\forall u^{\delta} A_{0}(x, u) \rightarrow B(x, y)\right) .
$$

Now define $C(x, y): \equiv\left(\forall u^{\delta} A_{0}(x, u) \rightarrow B(x, y)\right)$ and consider $C^{D}(x, y) \equiv \exists \underline{a} \forall \underline{b} C_{D}(\underline{a}, \underline{b}, x, y)$. Then

$$
\left(\forall x^{\rho} \exists y^{\tau}\left(\forall u^{\delta} A_{0}(x, u) \rightarrow B(x, y)\right)\right)^{D} \equiv \exists Y, \underline{A} \forall x, \underline{b} C_{D}(\underline{A}(x), \underline{b}, x, Y(x)) .
$$

By (the proof of) theorem 2.2 we obtain closed terms $t, \underline{s}$ such that

$$
\mathrm{HA}^{\omega}+\mathcal{P} \vdash \forall x, \underline{b} C_{D}(\underline{s}(x), \underline{b}, x, t(x))
$$

and hence

$$
\mathrm{HA}^{\omega}+\mathcal{P} \vdash \forall x \exists \underline{a} \forall \underline{b} C_{D}(\underline{a}, \underline{b}, x, t(x))
$$


By proposition 3.1 we get

$$
\mathrm{H}^{\omega} \vdash \forall x\left(C(x, t(x)) \leftrightarrow \exists \underline{a} \forall \underline{b} C_{D}(\underline{a}, \underline{b}, x, t(x))\right)
$$

and so

$$
\mathrm{H}^{\omega}+\mathcal{P} \vdash \forall x^{\rho}\left(\forall u^{\delta} A_{0}(x, u) \rightarrow B(x, t(x))\right) .
$$

The combination of negative translation and functional interpretation (ND) yields the following program extraction theorem for classical proofs (see e.g. [69]):

Theorem 3.3 (Program extraction by ND-interpretation). Let $\mathcal{P}$ be as before and $A_{0}\left(x^{\rho}, u^{\delta}\right)$, $B_{0}\left(x^{\rho}, y^{\tau}\right)$ be quantifier-free formulas containing only $x, u$ resp. $x, y$ free and $\rho, \delta, \tau$ are arbitrary types. Then the following rule holds:

$$
\left\{\begin{array}{l}
\mathrm{PA}^{\omega}+\mathrm{QF}-\mathrm{AC}+\mathcal{P} \vdash \forall x^{\rho}\left(\forall u^{\delta} A_{0}(x, u) \rightarrow \exists y^{\tau} B_{0}(x, y)\right) \\
\text { then one can extract closed terms s, } t \text { of } \mathrm{HA}^{\omega} \text { s.t. } \\
\mathrm{HA}^{\omega}+\mathcal{P} \vdash \forall x^{\rho}\left(A_{0}(x, s(x)) \rightarrow B_{0}(x, t(x))\right) .
\end{array}\right.
$$

Again we may have tuples of variables $\underline{x}, \underline{y}, \underline{u}$.

The previous result has as a corollary the so-called no-counterexample interpretation of PA in HA ${ }^{\omega}$ (and even $T$ ). This interpretation was developed by Kreisel $[76,77]$ prior to Gödel's publication of the functional interpretation and involves for its formulation only functionals of type degree $\leq 2$. Consider a sentence $A$ in the language of PA in prenex normal form

$$
A \equiv \exists x_{1} \forall y_{1} \ldots \exists x_{n} \forall y_{n} A_{0}\left(x_{1}, y_{1}, \ldots, x_{n}, y_{n}\right)
$$

and its Herbrand normal form (written with function quantifiers rather than new function symbols)

$$
A^{H} \equiv \forall h_{1}, \ldots, h_{n} \exists x_{1}, \ldots, x_{n} A_{0}\left(x_{1}, h_{1}\left(x_{1}\right), \ldots, x_{n}, h_{n}\left(x_{1}, \ldots, x_{n}\right)\right) .
$$

The no-counterexample interpretation (short: n.c.i.) of $A$ asks for functionals $\Phi_{1}, \ldots, \Phi_{n}$ realizing ' $\exists x_{1}, \ldots, x_{n}$ ' in $A^{H}$, i.e. (writing $\underline{h}$ instead of $h_{1}, \ldots, h_{n}$ )

$$
\forall \underline{h} A_{0}\left(\Phi_{1}(\underline{h}), h_{1}\left(\Phi_{1}(\underline{h})\right), \ldots, \Phi_{n}(\underline{h}), h_{n}\left(\Phi_{1}(\underline{h}), \ldots, \Phi_{n}(\underline{h})\right)\right) .
$$

We then write $\underline{\Phi}$ n.c.i. A.

Corollary 3.4. Let $A$ be a prenex sentence provable in $\mathrm{PA}$, then one can extract from a given proof closed terms $\underline{\Phi}$ of $\mathrm{HA}^{\omega}$, i.e.

$$
\mathrm{HA}^{\omega} \vdash \underline{\Phi} \text { n.c.i. A. }
$$

Proof: Modulo the canonical embedding of PA into $\mathrm{PA}^{\omega}$ the assumption implies that $\mathrm{PA}^{\omega} \vdash A$ and hence a-fortiori $\mathrm{PA}^{\omega} \vdash A^{H}$. The conclusion now follows from theorem 3.3.

By coding the tuple of number variables $x_{1}, \ldots, x_{n}$ into a single number variable $x$ and then searching for the least such $x$ satisfying $A^{H}$ it is clear that the mere truth of $A$ already implies the existence of computable (and hence continuous in the sense of the Baire space) functionals $\underline{\Phi}$ satisfying the no-counterexample interpretation of $A$. However, from a proof of $A$, the extraction procedure via functional interpretation will produce subrecursive functionals whose complexity depends on the proof principles used. Note also that the program extraction theorem 3.3 above applies to $y^{\tau}$ of arbitrary type $\tau$ so that (except for $\tau=0$ ) such a search is no longer possible.

While the no-counterexample interpretation is an easy consequence of Gödel's functional interpretation (combined with negative translation) it is complicated to establish it directly as a proof 
interpretation in its own right. This is caused by the bad behavior of the no-counterexample interpretation w.r.t. the modus ponens rule. In fact, the solution of the modus ponens under this interpretation cannot be carried out (uniformly) by functionals definable in $T$ but requires a use of bar recursion (of lowest type). While this treatment of the modus ponens pointwise stays within $T$, the type level of the primitive recursion needed to satisfy the n.c.i. of the conclusion $B$ will in general be higher than that for the input functionals from the n.c.i. of the premises $A$ and $A \rightarrow B$ ([60]). In particular, this approach does not give optimal results for fragments of $\mathrm{PA}^{\omega}$ with restricted induction while the route of proving the no-counterexample interpretation via functional interpretation does $([95,60])$. In fact, the original proof of the no-counterexample interpretation (based on a different description of the type-2 functionals in $T$ as the $\alpha\left(<\varepsilon_{0}\right)$-recursive functionals of type 2) as given in [76, 77] was based on the $\varepsilon$-substitution method developed in [1] and so rather on a form of cut-elimination than established as a proof interpretation. A proof by direct use of cut-elimination was given subsequently in [97]. In fact, [34] shows that the no-counterexample interpretation was clearly anticipated by Gödel's analysis of Gentzen's consistency proof for PA based on cut-elimination. Functional interpretation provides a modular way of constructing functionals satisfying the no-counterexample interpretation. As the latter can be viewed as a generalization of Herbrand's theorem it is natural to look also for an extraction algorithm based on functional interpretation of valid Herbrand disjunctions from proofs in classical logic. This was suggested already by $\mathrm{G}$. Kreisel in [81] and carried out finally in [26].

\section{A comparison of interpretations of $\Pi_{3}^{0} \rightarrow \Pi_{2}^{0}$}

In this section we discuss in detail the treatment of a special case of the modus ponens by functional interpretation (in particular when combined with negative translation) in comparison with other interpretations. The first non-trivial instance of the modus ponens in a context based on classical logic is the situation where a $\forall \exists$-sentence (say in the language of first order arithmetic PA, i.e. a socalled $\Pi_{2}^{0}$-sentence) $B$ is proved using a lemma $A$ of the slightly more complicated logical structure: $\forall \exists \forall$, i.e. $A \in \Pi_{3}^{0}$. We will see that only the combination of negative translation with functional interpretation applied to ineffective proofs of $A$ and $A \rightarrow B$ produces a satisfying computational realizer for $B$. In fact, the performance of functional interpretation on the negative fragment is so strong that applying first negative translation to the proof of $A \rightarrow B$ improves the situation even in the case where $A \rightarrow B$ is constructively proven (say in HA). Note that relative to intuitionistic arithmetic plus the Markov principle for numbers $(A \rightarrow B)^{\prime}$ is a stronger statement than $A \rightarrow B$.

This situation (formulated in suitable extensions of PA and $\mathrm{PA}^{\omega}$ by abstract classes of spaces, see below) already covers some important applications of functional interpretation in metric fixed point theory: here often the convergence towards 0 of some nonincreasing sequence $\left(t_{n}\right)$ of nonnegative real numbers (defined in various parameters involving the spaces and functions in question) towards 0 is proved using the Cauchy property of this or some related sequence $\left(s_{n}\right)$ of real numbers, i.e.

$$
\forall k \in \mathbb{N} \exists n \in \mathbb{N} \forall m \in \mathbb{N}\left(\left|s_{n}-\mathbb{R} s_{n+m}\right| \leq_{\mathbb{R}} 2^{-k}\right) \rightarrow \forall k \in \mathbb{N} \exists n \in \mathbb{N} \forall m \geq n\left(t_{m}<_{\mathbb{R}} 2^{-k}\right) .
$$

Using an appropriate representation of real numbers as Cauchy sequences of rational numbers with fixed rate of convergence one has $\leq_{\mathbb{R}} \in \Pi_{1}^{0}$ and $<_{\mathbb{R}} \in \Sigma_{1}^{0}$. Since the monotonicity of $\left(t_{n}\right)$ implies that $\forall m \geq n\left(t_{m}<_{\mathbb{R}} 2^{-k}\right)$ is equivalent to $t_{n}<_{\mathbb{R}} 2^{-k}$ this implication has the form $\Pi_{3}^{0} \rightarrow \Pi_{2}^{0}$. The problem now is to get an effective bound on ' $\exists n$ ' in the conclusion despite of the fact that there in general is none for ' $\exists n$ ' in the premise: e.g. suppose that $\left(s_{n}\right)$ too is a nonincreasing sequence of nonnegative real numbers. Then the Cauchy property always holds, but by E. Specker [101] there are simple primitive recursively computable such sequences for which there is no effective rate of convergence.

Consider sentences $A, B$ in the language of Peano arithmetic $\mathcal{L}(\mathrm{PA})$ having the following form

$$
A \equiv \forall x \exists y \forall z A_{0}(x, y, z), \quad B \equiv \forall u \exists v B_{0}(u, v),
$$


where $A_{0}, B_{0}$ are quantifier-free formulas (containing only the variables $x, y, z$ resp. $u, v$ ).

We will compare in which data (and with what complexity) the interpretations provide a witness function $\varphi$ for $B$, i.e.

$$
\forall u B_{0}(u, \varphi(u)) .
$$

The interpretations we consider are: Kleene realizability, modified realizability and functional interpretation as well as each of these interpretations combined with negative translation as a preprocessing step. The preprocessing step using negative translation in general is unavoidable when $A$ and/or $A \rightarrow B$ are given with ineffective proofs since all the interpretations mentioned do not apply to classical proofs. Since modified realizability is trivial on the result of the application of negative translation we, furthermore, discuss the use of the so-called Friedman/Dragalin $A$-translation (see below) as an intermediate step. Finally, we compare the result of applying functional interpretation with the no-counterexample interpretation.

\subsection{Kleene realizability of $\Pi_{3}^{0} \rightarrow \Pi_{2}^{0}$}

A Kleene realizer $e \in \mathbb{N}$ for $A \rightarrow B$ (short $e r(A \rightarrow B)$ ) is (a code $e \in \mathbb{N}$ of) a partial computable 2-place function mapping any code $f \in \mathbb{N}$ of a hypothetical partial recursive function satisfying the Kleene realizability of $A$, i.e.

$$
\forall x\left(\{f\}(x) \downarrow \wedge \forall z A_{0}(x,\{f\}(x), z)\right),
$$

and any number $u \in \mathbb{N}$ into a witness for ' $\exists v B_{0}(u, v)$ ', i.e.

$$
\text { e } r(A \rightarrow B): \equiv \forall f, u \in \mathbb{N}\left(\forall x\left(\{f\}(x) \downarrow \wedge \forall z A_{0}(x,\{f\}(x), z)\right) \rightarrow\{e\}(f, u) \downarrow \wedge B_{0}(u,\{e\}(f, u))\right) .
$$

So a given recursive witnessing function $\{f\}$ for ' $\exists y$ ' in the premise $A$ is translated into a recursive witnessing function $\lambda u .\{e\}(f, u)$ for ' $\exists v$ ' in the conclusion $B$.

This requirement to have a recursive witness for $A \in \Pi_{3}^{0}$ can, in general, only be expected to be satisfiable if $A$ has a constructive proof (e.g. in Heyting arithmetic HA) as E. Specker's example discussed above shows. For an even simpler counterexample from recursion theory consider (here $T$ denotes the standard Kleene $T$-predicate) the following:

$$
A: \equiv \forall x \exists y \forall z(T(x, x, y) \vee \neg T(x, x, z))
$$

is provable already in plain (classical) logic but there is not even a recursive bound on ' $\exists y$ ' (in $x$ ) as such a bound would provide a decision procedure for the special halting problem $\{x: \exists y T(x, x, y)\}$.

\subsection{Modified realizability interpretation of $\Pi_{3}^{0} \rightarrow \Pi_{2}^{0}$}

The modified realizability interpretation of $A \rightarrow B$ is satisfied by any functional $\Phi: \mathbb{N}^{\mathbb{N}} \times \mathbb{N} \rightarrow \mathbb{N}$ such that

$$
\forall f \in \mathbb{N}^{\mathbb{N}}, u \in \mathbb{N}\left(\forall x, z A_{0}(x, f(x), z) \rightarrow B_{0}(u, \Phi(f, u))\right) .
$$

We then write $\Phi m r(A \rightarrow B)$.

In difference to Kleene realizability the modified realizability interpretation of a (HA-)proof of $A \rightarrow B$ will provide a subrecursive functional $\Phi$ that is primitive recursive in the sense of Gödel's $T$ (and of much lower complexity for appropriate fragments of HA). Moreover, $\Phi$ applies to any function $f$ and not just to computable ones. However, the weakness of this interpretation for ineffective premises $A$ is similar to that of Kleene realizability: if there is no computable $f$ such that $\forall x, z A_{0}(x, f(x), z)$ then the modified realizability interpretation does not yield an effective witness for the conclusion $B$. 


\subsection{Functional interpretation of $\Pi_{3}^{0} \rightarrow \Pi_{2}^{0}$}

The functional interpretation $(A \rightarrow B)^{D}$ of $A \rightarrow B$ is given by

$$
\exists V, Z, X \forall f, u\left(A_{0}(X(f, u), f(X(f, u)), Z(f, u)) \rightarrow B_{0}(u, V(f, u))\right) .
$$

So a realization of the functional interpretation of $A \rightarrow B$ is a triple of functionals $\varphi_{1}, \varphi_{2}, \varphi_{3}$ such that

$$
\forall f, u\left(A_{0}\left(\varphi_{1}(f, u), f\left(\varphi_{1}(f, u)\right), \varphi_{2}(f, u)\right) \rightarrow B_{0}\left(u, \varphi_{3}(f, u)\right)\right) .
$$

We then write $\underline{\varphi} D(A \rightarrow B)$.

As in the case of the modified realizability, any function $f$ realizing $A$, i.e.

$$
(*) \forall x, z A_{0}(x, f(x), z),
$$

can be used to perform the modus ponens to obtain $\lambda u . \varphi_{3}(f, u)$ as a witness function for the conclusion $B$. This time, however, instead of $(*)$ for each $u$ the weaker requirement on $f$

$$
(*)^{-} A_{0}\left(\varphi_{1}(f, u), f\left(\varphi_{1}(f, u)\right), \varphi_{2}(f, u)\right)
$$

suffices. For possible uses of this in number theory, also for $B \in \Pi_{3}^{0}$, where a similar weakening of the premise $A$ is possible, see [84] (this paper contains an extensive discussion of the treatment of $\left(\Pi_{3}^{0} \rightarrow \Pi_{3}^{0}\right)$-implications by functional interpretation).

Suppose now that we have a (subrecursive) functional $\varphi_{0}$ satisfying

$$
\text { (\%) } \forall x, g A_{0}\left(x, \varphi_{0}(x, g), g\left(\varphi_{0}(x, g)\right)\right) \text {, }
$$

i.e. the no-counterexample interpretation of $A$ (which - in this case - coincides with the functional interpretation $\left(A^{\prime}\right)^{D}$ of the negative translation $A^{\prime}$ of $A$ using the stability of $\left.A_{0}\right)$. If we then are able to solve the system of equations

$$
x=\varphi_{1}(f, u), \varphi_{0}(x, g)=f\left(\varphi_{1}(f, u)\right), g\left(\varphi_{0}(x, g)\right)=\varphi_{2}(f, u)
$$

for $f, x, g$ in $u$ then we are able to solve the modus ponens and obtain $B_{0}\left(u, \varphi_{3}(f, u)\right)$ for this $f$. Such a solution can indeed by constructed by means of Spector's aforementioned bar recursive functionals. In the case at hand, only bar recursion $B_{0,1}$ at the lowest type is needed which, applied to arguments in Gödel's $T$, stays within $T$ (although $T+B_{0,1}$ goes beyond $T$ ) but - in general increases the complexity of the arguments: if the arguments only use recursion of type level $n$, the result of applying $B_{0,1}$ to them will produce a functional whose $T$-definition may need recursion of level $n+1$. For details on all this see [60] and the literature cited there (in particular [51] and [98]). For the special case of the modus ponens problem just discussed, where instead of

$$
\forall x \exists y \forall z A_{0}(x, y, z) \rightarrow \forall u \exists v B_{0}(u, v),
$$

we can prove the stronger form

$$
(+) \forall u\left(\exists y \forall z A_{0}(u, y, z) \rightarrow \exists v B_{0}(u, v)\right),
$$

the use of bar recursion can be avoided: the functional interpretation $(+)^{D}$ of $(+)$ provides us with functions $t, s$ such that

$$
\forall u, y\left(A_{0}(u, y, t(u, y)) \rightarrow B_{0}(u, s(u, y))\right)
$$

Then substituting $\varphi_{0}(u, \lambda y . t(u, y))$ for $y$ and using $(\%)$ with $g:=\lambda y . t(x, y)$ yields

$$
p(u):=s\left(u, \varphi_{0}(u, \lambda y . t(u, y))\right)
$$


as a witness function for $B$, i.e. $\forall u B_{0}(u, p(u))$.

Note that this special case does not lead to an improvement of the situation for Kleene or modified realizability above since both interpretations fail to produce a witness $t(u, y)$ for ' $\forall z$ ' in the premise.

The significance of the improvement achieved by functional interpretation depends on whether a nontrivial effective $\varphi_{0}$ satisfying $(\%)$ can in fact be extracted from a classical proof of $A$. In section 4.5 we will show that this can be achieved by applying negative translation followed by functional interpretation to the proof of $A$.

For related discussions of the weakness of a constructive (BHK-style) interpretation of implications $\Pi_{3}^{0} \rightarrow \Pi_{3}^{0}$ and $\Pi_{3}^{0} \rightarrow \Pi_{2}^{0}$ as spelled out by (modified) realizability compared to the strengthened interpretation provided by functional interpretation see Kreisel-Macintyre [84] and Kreisel [82].

\subsection{Negative translation followed by Kleene realizability resp. modified realizability of $\Pi_{3}^{0} \rightarrow \Pi_{2}^{0}$}

The negative translations of $A, A \rightarrow B$ and $B$ are over HA equivalent to

$$
\begin{gathered}
\forall x \neg \forall y \neg \forall z A_{0}(x, y, z), \\
\forall x \neg \forall y \neg \forall z A_{0}(x, y, z) \rightarrow \forall u \neg \forall v \neg B_{0}(u, v)
\end{gathered}
$$

and

$$
\forall u \neg \forall v \neg B_{0}(u, v)
$$

and hence to so-called $\exists$-free formulas. However, the Kleene realizability interpretation $x r F$ of an $\exists$-free formula $F$ is just the formula itself and does not depend at all on $x$ (e.g. we may take $x:=0$ ). Similarly, for modified realizability where now the empty tuple is the realizer.

In order to obtain from

$$
\forall u \neg \forall v \neg B_{0}(u, v)
$$

a formula for which realizability interpretations are nontrivial we must be able to convert this back to the original conclusion

$$
\forall u \exists v B_{0}(u, v)
$$

which amounts to an application of the (rule version of the) Markov principle $\mathrm{M}^{0}$ for numbers

$$
\mathrm{M}^{0}: \neg \forall x \neg F_{0}(x) \rightarrow \exists x F_{0}(x),
$$

where $F_{0}(x)$ is a quantifier-free formula (which may contain additional parameters). However, the modified realizability interpretation of $\mathrm{M}^{0}$ has (for suitable $F_{0}$ ) no effective solution ([79]): take $F_{0}(x): \equiv T(a, a, x)$, where $T$ is Kleene's $T$-predicate. Then

$$
f m r \forall a(\neg \forall x \neg T(a, a, x) \rightarrow \exists x T(a, a, x)) \leftrightarrow \forall a(\neg \forall x \neg T(a, a, x) \rightarrow T(a, a, f(a))) .
$$

By the undecidability of the special halting problem, however, there is no computable $f$ with this property. For Kleene realizability the situation is different but equally unfortunate: whereas modified realizability asks for a total function $f$, Kleene realizability only requires a partial recursive function which exists by trivial unbounded search

$$
f(a):=\left\{\begin{array}{l}
\text { least } x \text { with } T(a, a, x), \text { if } \exists x T(a, a, x) \\
\text { undefined, otherwise. }
\end{array}\right.
$$

But although $\mathrm{M}^{0}$ is Kleene realizable in this way, this does not help for the modus ponens problem at hand since rather than analyzing the proof of $B$ (via the proofs of $A$ and $A \rightarrow B$ ) just an unbounded search is performed which totally disregards the proof of $B$ (but only uses the truth of $B$ for the termination). In particular, no subrecursive complexity information is obtained. 


\subsection{Negative translation followed by functional interpretation of $\Pi_{3}^{0} \rightarrow \Pi_{2}^{0}$}

The functional interpretations $\left(A^{\prime}\right)^{D}$ and $\left((A \rightarrow B)^{\prime}\right)^{D}$ of the negative translations $A^{\prime}$ and $(A \rightarrow B)^{\prime}$ of $A$ and $A \rightarrow B$ are equivalent over $\mathrm{HA}^{\omega}$ to

$$
\text { (1) } \exists Y \forall x, g A_{0}(x, Y(x, g), g(Y(x, g)))
$$

and

$$
\begin{aligned}
& \exists X, G, V \forall Y, u \\
& \left(A_{0}\left(X(Y, u), Y(X(Y, u), G(Y, u)),(G(Y, u))(Y(X(Y, u), G(Y, u))) \rightarrow B_{0}(u, V(Y, u))\right)\right.
\end{aligned}
$$

and $\left(B^{\prime}\right)^{D}$ is just (equivalent over $\mathrm{HA}^{\omega}$ to)

$$
\exists f \forall u B_{0}(u, f(u)) .
$$

If we throw away the information provided by $X, G$, the (then 'partial') functional interpretation of $\left((A \rightarrow B)^{\prime}\right)^{D}$ simplifies to

$$
\text { (2) } \exists V \forall Y, u\left(\forall x, g A_{0}(x, Y(x, g), g(Y(x, g))) \rightarrow B_{0}(u, V(Y, u))\right)
$$

From a realizer $\varphi_{0}$ for ' $\exists Y$ ' in (1) and a realizer $\Phi$ for ' $\exists V$ ' in (2) we now obtain a realizer for $B$ just by application, i.e. for $f(u):=\Phi\left(\varphi_{0}, u\right)$ we have

$$
\forall u B_{0}(u, f(u))
$$

Let us finally indicate how such a solution $\varphi_{0}$ can be obtained for the ineffective Cauchy property of a nonincreasing sequence of nonnegative and (for simplicity) rational numbers $\left(s_{n}\right):$ define $\varphi(0, g):=$ $0, \varphi(k+1, g):=\varphi(k, g)+g(\varphi(k, g))$. Then (for $N \geq s_{0}$ ) one can show that

$$
\text { (3) } \exists n \leq \varphi\left(N 2^{k}, g\right)\left(\left|s_{n}-s_{n+g(n)}\right| \leq_{\mathbb{Q}} 2^{-k}\right)
$$

and hence $\varphi_{0}(k, g)$ can be defined as the least such $n$. In fact, $n$ can be taken as $n:=\varphi(i, g)$ for a suitable $i<N \cdot 2^{k}$. Note that - in contrast to $\varphi_{0}$ - the bound $\varphi$ depends on $\left(s_{n}\right)$ only via $N$.

\subsection{Negative translation followed by $A$-translation and modified realiz- ability of $\Pi_{3}^{0} \rightarrow \Pi_{2}^{0}$}

H. Friedman [23] and A. Dragalin [20] independently developed a so-called $A$-translation of a formula $F$ where every prime formula $P$ (including $\perp$ ) in $F$ gets replaced by $P \vee A$ (here $\neg A$ must be defined as $A \rightarrow \perp)$. Applying this technique to $A: \equiv \exists v^{0} B_{0}(u, v)$ one can convert an HA-proof of $\neg \forall v \neg B_{0}(u, v)$ (resulting from negative translation) into an HA-proof of $\exists v B_{0}(u, v)$ to which then modified realizability can be applied. This does provide effective realizers from classical proofs of $B$ (via classical proofs of $A$ and $A \rightarrow B$ ) but the complexity usually is not optimal: as we saw in section 4.5 the negative translation of the Cauchy-property of $\left(s_{n}\right)$ has a functional interpretation using only primitive recursion at the lowest type 0 . In contrast to this, the $\exists v B_{0}(u, v)$-translation of the negative translation apparently requires primitive recursion at type 1 (sufficient to define the Ackermann function) for its modified realizability interpretation (see [69]). ${ }^{2}$ Strangely enough, functional interpretation of the $\exists v B_{0}(u, v)$-translation (though that step actually is not necessary by our discussion above) again only uses primitive recursion of type 0 . So here we have a statement whose modified realizability seems to require more complicated functionals than its functional interpretation. However, this is not really surprising: since modified realizability gives a much weaker

\footnotetext{
${ }^{2}$ An optimized Kripke-style version of the $A$-translation which sometimes, e.g. in this case, gives a solution of the right complexity was developed in [18].
} 
treatment of implications it is usually harder to satisfy when applied to an implication whose premise again is an implication but whose conclusion is simple. It is exactly this situation the $A$-translation applied to the result of the negative translation leads into. Refined versions of $A$-translation which use that the result of applying negative translation can be verified in a context based on minimal logic are even more complicated here: one now only has to replace $\perp$ by $\exists v^{0} B_{0}$ (but not the prime formula $P: \equiv\left|s_{n}-\mathbb{Q} s_{n+g(n)}\right| \leq_{\mathbb{Q}} 2^{-k}$ by $\left.P \vee \exists v^{0} B_{0}\right)$, but one no longer can disregard the double negation in front of $P$ which results from the 'official' negative translation (which we tacitly dropped in our intuitionistic context of $\mathrm{HA}^{\omega}$ ). One should also note that the approach via any form of $A$ translation interprets $A$ based on the conclusion $B$ of the proof which uses $A$ whereas functional interpretation is fully modular in the sense that $A$ in interpreted in a way sufficient for any use of it in any proof of any conclusion.

\subsection{Discussion of the results of the comparison}

As the treatment of $A \rightarrow B$ with $A \in \Pi_{3}^{0}$ and $B \in \Pi_{2}^{0}$ shows, the combination of functional interpretation with negative translation gives the most successful interpretation of this modus ponens. This even is the case when $A \rightarrow B$ is already proved constructively so that negative translation would not be necessary to be applied to the proof of $A \rightarrow B$. Only in the special case where one can strengthen $A \rightarrow B$ to ${ }^{3}$

$$
(+) \forall u\left(\exists y \forall z A_{0}(u, y, z) \rightarrow \exists v B_{0}(u, v)\right)
$$

the direct functional interpretation of a constructive proof of $A \rightarrow B$ provides a similarly strong result whereas in the general case bar recursion (at the lowest level) would be needed which is avoided by the use of negative translation.

The direct functional interpretation of $A \rightarrow B$ (i.e. without negative translation as a pre-processing step) actually coincides with the no-counterexample interpretation of the following prenex normal form $(A \rightarrow B)^{p r}$ of $A \rightarrow B$ :

$$
\forall u \exists x \forall y \exists z, v\left(A_{0}(x, y, z) \rightarrow B_{0}(u, v)\right),
$$

whereas the no-counterexample interpretation of $A$ coincides with the functional interpretation of the negative translation $A^{\prime}$ of $A$. So in order to perform the modus ponens under the no-counterexample interpretation one needs exactly the same use of bar recursion $B_{0,1}$ as discussed in 4.3 above (for a detailed discussion see [60]). For the special case $(+)$, the no-counterexample interpretation yields results as strong as the combination of negative translation with functional interpretation but to verify the soundness of the former for a given system one either has to prove the soundness of the latter or to apply a suitable form of $\varepsilon$-substitution or cut-elimination $([76,77,97])$ which destroys the modularity of the interpretation.

\section{Comparison between $\left(A^{\prime}\right)^{D}$ and other (classical) $\exists \forall$-normal forms:}

We now further illuminate the good behavior of the combination $\left(A^{\prime}\right)^{D}$ of negative translation $A^{\prime}$ and functional interpretation $A^{D}$ for proofs based on classical logic: by the application of functional interpretation, clearly $\left(A^{\prime}\right)^{D}$ has the form $\exists \underline{u} \forall \underline{v}\left(A^{\prime}\right)_{D}(\underline{u}, \underline{v})$, where $\left(A^{\prime}\right)_{D}$ is quantifier-free and $\underline{u}, \underline{v}$ are tuples of variables for functionals of finite types (where the length and the types only depend on the logical structure of $A$ ). Since we work with classical logic we may assume that $A$ is given in prenex normal form (with quantifier-free $A_{0}$ )

$$
\forall x_{1} \exists y_{1} \ldots \forall x_{n} \exists y_{n} A_{0}\left(x_{1}, y_{1}, \ldots, x_{n}, y_{n}\right) .
$$

As in the section above we only treat the case where $A$ is arithmetical, i.e. where $x_{1}, y_{1}, \ldots, x_{n}, y_{n}$ have the type 0 of natural numbers. However, things also generalize to the case where these variables may have arbitrary types.

\footnotetext{
${ }^{3}$ In $A_{0}$ one can also allow $r(u)$ instead of $u$ for some term $r$.
} 
The most well-known $\exists \forall$ normal form of $A$ results by forming the Skolem normal form which in the presence of function variables can be written as

$$
A^{S}: \equiv \exists f_{1}, \ldots, f_{n} \forall x_{1}, \ldots, x_{n} A_{0}\left(x_{1}, f_{1}\left(x_{1}\right), \ldots, x_{n}, f_{n}\left(x_{1}, \ldots, x_{n}\right)\right) .
$$

While the implication $A^{S} \rightarrow A$ is trivial, the converse implication (and hence the equivalence between $A$ and $A^{S}$ ) can be justified using the following arithmetical axiom of choice for numbers ${ }^{4}$

$$
\mathrm{AC}_{a r}^{0,0}: \forall \underline{x} \exists y A_{a r}(\underline{x}, y) \rightarrow \exists f \forall \underline{x} A_{a r}(\underline{x}, f(\underline{x})),
$$

where $\underline{x}=x_{1}, \ldots, x_{k}, y$ are variables for numbers and $A_{a r}$ is an arithmetical formula.

Compared to $A^{S}$, the no-counterexample interpretation first forms the dual of the Skolem normal form, the so-called Herbrand normal form

$$
A^{H}: \equiv \forall g_{1}, \ldots, g_{n} \exists y_{1}, \ldots, y_{n} A_{0}\left(g_{1}, y_{1}, g_{2}\left(y_{1}\right), y_{2}, \ldots, g_{n}\left(y_{1}, \ldots, y_{n-1}\right), y_{n}\right),
$$

and then applies quantifier-free choice

$$
\text { QF-AC : } \forall \underline{x} \exists \underline{y} A_{0}(\underline{x}, \underline{y}) \rightarrow \exists \underline{Y} \forall \underline{x} A_{0}(\underline{x}, \underline{Y}(\underline{x})) \quad\left(A_{0}\right. \text { quantifier-free) }
$$

(though with function arguments $\underline{x}$ ) to obtain

$$
A^{n . c . i .}: \equiv \exists Y_{1}, \ldots, Y_{n} \forall \underline{g} A_{0}\left(g_{1}, Y_{1}(\underline{g}), g_{2}\left(Y_{1}(\underline{g})\right), Y_{2}(\underline{g}), \ldots, g_{n}\left(Y_{1}(\underline{g}), \ldots, Y_{n-1}(\underline{g})\right), Y_{n}(\underline{g})\right) .
$$

Since the implication $A \rightarrow A^{H}$ is trivial, the implication $A \rightarrow A^{\text {n.c.i. }}$ only requires quantifier-free choice QF-AC. However, the implication $A^{H} \rightarrow A$ and hence $A^{\text {n.c.i. }} \rightarrow A$ again can be justified only using arithmetical choice $\mathrm{AC}_{a r}^{0,0}$. The use of $\mathrm{AC}_{a r}^{0,0}$ to prove $A \rightarrow A^{S}$ and $A^{H} \rightarrow A$ is unavoidable since both schemas (when stated for arbitrary arithmetical $A$ ) in fact imply $\mathrm{AC}_{a r}^{0,0}$ as is easy to show. In contrast to this, the relationship between $A$ and $\left(A^{\prime}\right)^{D}$ is much closer than the relationship between $A$ and $A^{S}$ as well as $A$ and $A^{n . c . i}$ respectively: instead of arithmetical choice (for numbers) only quantifier-free choice (though for higher types) is needed to establish the equivalence:

Proposition 4.1. 1) (G. Kreisel [78]) Let $A$ be an arbitrary formula of the language of $\mathrm{PA}^{\omega}$. Then

$$
\mathrm{PA}^{\omega}+\mathrm{QF}-\mathrm{AC} \vdash A \leftrightarrow\left(A^{\prime}\right)^{D}
$$

2) Let $A$ be in prenex normal form and arithmetical ${ }^{5}$

$$
\mathrm{HA}^{\omega} \vdash A^{S} \rightarrow\left(A^{\prime}\right)^{D} \rightarrow A^{n . c . i} .
$$

In general, both implications cannot be reversed over $\mathrm{PA}^{\omega}+\mathrm{QF}-\mathrm{AC}$.

Proof: 1) See [108](3.5.13) with a correction in [69]. 2) is proved in [69].

As we saw above, the Skolem normal form $A^{S}$ is too strong to be useful for proofs based on classical logic as already $\Pi_{3}^{0}$-lemmas in general will not have a computable Skolem function. The no-counterexample interpretation weakens the requirement sufficiently to allow effective (and even subrecursive) solutions but - when applied to some prenex normal form of a sentence $\Pi_{3}^{0} \rightarrow \Pi_{2}^{0}-$ is too weak for a simple treatment of the modus ponens (but has to use some amount of bar recursion). $\left(A^{\prime}\right)^{D}$ provides the right balance between the two extreme interpretations $A^{S}$ and $A^{\text {n.c.i. }}$. The price to be paid for this is that with an increasing number of quantifier alternations the degrees of the types of the functionals increase (i.e. higher and higher function spaces are needed) whereas for $A^{S}$ resp. $A^{\text {n.c.i. }}$ variables of type degree 1 resp. 2 are sufficient (i.e. only the arity but not the degree

\footnotetext{
${ }^{4}$ Since one can avoid choice by choosing the least number satisfying the property this schema corresponds, viewed from the perspective of set theory, to (arithmetical) comprehension and is not a proper form of choice.

${ }^{5}$ The result also holds for general prenex $A$ with the definitions of $A^{S}$ and $A^{n . c . i .}$ extended in the obvious way.
} 
of the type increases).

In practice, $\left(A^{\prime}\right)^{D}$ in many cases coincides with the no-counterexample interpretation due to possible strengthenings $(+)$ of implications $\Pi_{3}^{0} \rightarrow \Pi_{2}^{0}$ as discussed in 4.3 above by which $\Pi_{3}^{0} \rightarrow \Pi_{2}^{0}$ reduces from $\Sigma_{3}^{0}$ to $\Pi_{2}^{0}$.

In general, $\left(A^{\prime}\right)^{D}$ and $A^{\text {n.c.i. }}$ coincide as long $A$ is in $\Pi_{3}^{0}$ (as the Cauchy property of bounded monotone sequences) but differ from $A \in \Sigma_{3}^{0}$ on. Let us discuss this a bit further but first revisit the solution for $\left(A^{\prime}\right)^{D}$ of the Cauchy property $A$ for monotone sequences $\left(s_{n}\right)$ in $[0, \infty) \cap \mathbb{Q}$ given in (3) above. The bound also holds for nonincreasing sequences of real numbers $\left(s_{n}\right)$ in $[0, \infty)$ and can be rewritten in the following form (where $[n ; n+m]:=\{n, n+1, n+2, \ldots, n+m\}$ )

$$
\text { (4) } \exists n \leq \varphi\left(N 2^{k}, g\right) \forall i, j \in[n ; n+g(n)]\left(\left|s_{i}-s_{j}\right| \leq_{\mathbb{R}} 2^{-k}\right) \text {. }
$$

Again there actually exists such an $n$ of the form $n=\varphi(i, g)$ for a suitable $i<N \cdot 2^{k}$.

(4) yields (a quantitative form of) the so-called 'finite convergence principle' formulated recently by T. Tao $([106,107])$ :

Corollary 4.2. For all $k, N \in \mathbb{N}, g \in \mathbb{N}^{\mathbb{N}}$ there exists an $M \in \mathbb{N}$ such that for all nonincreasing finite sequences $0 \leq s_{M} \leq \ldots \leq s_{0} \leq N$ of length $M+1$ in $[0, N]$ there exists an $n \in \mathbb{N}$ with

$$
n+g(n) \leq M \wedge \forall i, j \in[n ; n+g(n)]\left(\left|s_{i}-s_{j}\right| \leq 2^{-k}\right) .
$$

Moreover, we can compute $M$ as $M:=\varphi\left(N 2^{k}, g\right)$ with $\varphi$ as above.

The fact that the bound in (4) is independent from the sequence $\left(s_{n}\right)$ in $[0, N]$ (which is crucially used in the above corollary) is an instance of a general phenomenon which can be established via a so-called monotone variant of functional interpretation due to [58] and discussed in the next section (note that $[0, N]^{\infty}$ is a compact metric space w.r.t. the product metric).

We conclude this section by briefly mentioning another principle $A$ recently discussed by Tao for which $A^{\text {n.c.i. }}$ and $\left(A^{\prime}\right)^{D}$ radically differ since it no longer is of the form $\forall \exists \forall$ (unless a finite collection principle is used which is as strong as the principle $A$ itself): the infinitary pigeonhole principle (IPP) is defined as

$$
\text { (IPP): } \forall n \in \mathbb{N} \forall f: \mathbb{N} \rightarrow C_{n} \exists i \leq n \forall k \in \mathbb{N} \exists m \geq k(f(m)=i),
$$

where $C_{n}:=\{0,1, \ldots, n\}$.

The Herbrand normal form of (IPP) is

$$
(\mathrm{IPP})^{H} \equiv \forall n \in \mathbb{N} \forall f: \mathbb{N} \rightarrow C_{n} \forall F: C_{n} \rightarrow \mathbb{N} \exists i \leq n \exists m \geq F(i)(f(m)=i)
$$

which gives rise to the following computationally almost trivial solution for the n.c.i. of IPP:

$$
M(n, f, F):=\max \{F(i): i \leq n\} \text { and } I(n, f, F):=f(M(n, f, F))
$$

are realizers for ' $\exists m$ ' and ' $\exists i$ ' in (IPP $)^{H}$. By contrast, the $\forall \exists$-form of $\left(A^{\prime}\right)^{D}$ is arrived at as follows

$$
\begin{aligned}
& (\mathrm{IPP}) \stackrel{\mathrm{QF}-\mathrm{AC}}{\Leftrightarrow} \\
& \forall n \in \mathbb{N} \forall f: \mathbb{N} \rightarrow C_{n} \exists i \leq n \exists g: \mathbb{N} \rightarrow \mathbb{N} \forall k \in \mathbb{N}(g(k) \geq k \wedge f(g(k))=i)^{\mathrm{QF}-\mathrm{AC}} \\
& \forall n \in \mathbb{N} \forall f: \mathbb{N} \rightarrow C_{n} \forall K: C_{n} \times \mathbb{N}^{\mathbb{N}} \rightarrow \mathbb{N} \exists i \leq n \exists g: \mathbb{N} \rightarrow \mathbb{N} \\
& \qquad(g(K(i, g)) \geq K(i, g) \wedge f(g(K(i, g)))=i)
\end{aligned}
$$

and requires (highly nontrivial) functionals $I(n, f, K)$ and $G(n, f, K)$ realizing ' $\exists i$ ' and ' $\exists g$ ' to solve $\left(A^{\prime}\right)^{D}$ (see [94] and [69] for details). In fact, since (IPP) implies (over weak base systems) the 
induction axiom for $\Sigma_{1}^{0}$-formulas (whereas itself follows from $\Sigma_{2}^{0}$-induction), the computational contribution of a use of (IPP) in a proof can be of arbitrary primitive recursive (in the sense of Kleene) complexity which is not properly accounted for by the simple n.c.i.-solution above but only by the solution of its ND-interpretation. By the aforementioned monotone variant of functional interpretation (to be discussed below) one obtains a bound on ' $\exists g$ ' which is independent from the coloring $f$ and which - combined with a uniform continuity argument - yields a quantitative version of the 'finitary' version of (IPP) introduced by Tao $([106,107])$. It, therefore, seems to be the case that the program of so-called 'hard analysis' as advocated in [106] is closely related to carrying out the monotone functional interpretation of proofs in analysis. In fact, the results discussed in the next section have recently been applied by Avigad et al. [4] to obtain the type of uniform quantitative analysis in ergodic theory discussed in Tao [106] and have been used already in Tao [107].

\section{Extraction of effective uniform bounds in analysis}

Since the 90's proof theoretic methods based on specially designed variants and extensions of functional interpretations have been used extensively for the 'unwinding' (G. Kreisel) of prima facie ineffective proofs in analysis, functional analysis and - most recently - in geodesic geometry. This approach, also called 'proof mining', has led to a number of new effective quantitative results but also to new qualitative results on the independence of solutions from certain parameters (uniformity results). The following papers in analysis use directly such techniques or use results obtained by these techniques or have been guided by general logical metatheorems which were established using functional interpretations: $[4,10,11,12,13,14,25,57,61,62,63,65,70,71,72,73,74,88]$. For surveys see $[67,68]$ and - though covering only results up to 2002 - [74] which explains in detail general aspects of applying functional interpretation to analysis. Much more information can be found in the forthcoming book [69]. [30] uses functional interpretation (though mostly in the form of n.c.i.) to analyze the proof of van der Waerden's theorem given by Furstenberg and Weiss based on topological dynamics.

In recent years, general logical metatheorems based on functional interpretations have been proved which for large classes of proofs and theorems guarantee the extractability of effective and strongly uniform bounds: $[28,56,64,66,87]$. This shows that the concrete applications are not 'ad hoc' and so meet the critique expressed in [21] of early stages of the unwinding program (see also [90] for a discussion of 'unwinding' in general).

In this section we sketch some of these metatheorems but for details refer to [56, 64, 28]). We state one concrete application in analysis but otherwise point to the papers listed above.

The most important variant of functional interpretation for these applications is the monotone functional interpretation (MD) which was introduced in [58]. It is like ordinary functional interpretation except that the statement in the soundness theorem for functional interpretation is replaced by

$$
(+) \exists \underline{x}\left(\underline{t}^{*} \gtrsim \underline{x} \wedge \forall \underline{a}, \underline{y} A_{D}(\underline{x}(\underline{a}), \underline{y}, \underline{a})\right)
$$

for suitable closed terms $\underline{t}^{*}$, where $\gtrsim$ is a suitable hereditarily defined majorization relation between functionals of type $\rho\left(\underline{t}^{*} \gtrsim \underline{x}\right.$ is to be understood coordinatewise). E.g. we may take W.A. Howard's [50] relation of majorizability or (which sometimes is more suitable) the following variant ('strong majorizability') due to M. Bezem ([5]):

$$
\left\{\begin{array}{l}
x^{*} \gtrsim_{0} x: \equiv x^{*} \geq x, \\
x^{*} \gtrsim_{\rho \rightarrow \tau} x: \equiv \forall y^{*}, y\left(y^{*} \gtrsim_{\rho} y \rightarrow x^{*} y^{*} \gtrsim_{\tau} x^{*} y \wedge x^{*} y^{*} \gtrsim_{\tau} x y\right) .
\end{array}\right.
$$

We then say that $\underline{t}^{*}$ satisfies the monotone functional interpretation of $A$. The soundness proof for the monotone functional interpretation proceeds by establishing $(+)$ by induction on the proof. It is similar to the usual soundness proof combined with some easy majorization arguments. In fact, 
the construction of the terms $\underline{t}^{*}$ is much simpler than the construction of $\underline{t}$ in the usual functional interpretation ([58]) which can be bypassed.

In the following, $\Delta$ is any set of sentences

$$
\forall a \exists b \leq r(a) \forall c F_{0}(a, b, c),
$$

where $a, b, c$ may have arbitrary types, $r$ is a closed term, $\leq$ is defined pointwise and $F_{0}$ is a quantifierfree formula. $\tilde{\Delta}$ consists of the Skolem normal forms

$$
\exists B \leq r \forall a, c F_{0}(a, B(a), c)
$$

of these sentences. 'NMD' denotes the combination of negative translation with monotone functional interpretation.

Theorem 5.1 (Uniform bound extraction [55, 58]).

Let $A_{0}\left(x^{1}, y^{\rho}, z^{\tau}\right)$ be a quantifier-free formula of $\mathcal{L}\left(\mathrm{PA}^{\omega}\right)$ containing only $x, y, z$ as free variables, $\operatorname{deg}(\tau) \leq 2$ and $s$ be a closed term. Then

$$
\left\{\begin{array}{l}
\mathrm{PA}^{\omega}+\mathrm{QF}-\mathrm{AC}+\Delta \vdash \forall x^{1} \forall y \leq_{\rho} s x \exists z^{\tau} A_{0}(x, y, z) \\
\Rightarrow \mathrm{NMD} \text { extracts a closed term } t \text { of } \mathrm{HA}^{\omega} \text { such that } \\
\mathrm{HA}^{\omega}+\tilde{\Delta} \vdash \forall x^{1} \forall y \leq_{\rho} s x \exists z \leq_{\tau} t x A_{0}(x, y, z) .
\end{array}\right.
$$

In particular, if $\mathcal{S}^{\omega} \models \Delta$, then the conclusion holds in $\mathcal{S}^{\omega}$. The result also applies to tuples of variables.

Remark 5.2. The above theorem also has a version which is more in the spirit of the original foundational aims of functional interpretation for consistency proofs: if the premise is provable in $\mathrm{PA}^{\omega}+\mathrm{QF}-\mathrm{AC}$ in the form ${ }^{6}$

$$
\Delta \rightarrow \forall x^{1} \forall y \leq_{\rho} s x \exists z^{\tau} A_{0}(x, y, z)
$$

and $\Delta$ is of the (w.r.t. the types) more restricted form

$$
\forall a^{1} \exists b \leq_{1} r(a) \forall c^{0} F_{0}(a, b, c)
$$

then the verification of the extracted bound can carried out using only the following approximate $(\varepsilon)$-form

$$
\Delta_{\varepsilon}: \equiv \forall a^{1}, c^{0} \exists b \leq_{1} r(a) \forall \tilde{c} \leq c F_{0}(a, b, c)
$$

of $\Delta$ which for many ineffective principles $\Delta$ has a simple constructive proof. E.g. the well-known binary ('weak') König's lemma WKL, which allows one to carry out many ineffective proofs in analysis and algebra ([100]), can be written as such a special axiom $\Delta$ whose $\varepsilon$-version is trivial. This yields a relative consistency proof of the part of mathematics that follows over $\mathrm{PA}^{\omega}+\mathrm{QF}-\mathrm{AC}$ from WKL relative to $\mathrm{HA}^{\omega}$ and so - by Gödel's [37] - relative to T. This has been carried out not only for $\mathrm{PA}^{\omega}$ but also for numerous fragments in [55, 59].

For a related so-called bounded functional interpretation see [22].

Using the so-called standard representation of complete separable ('Polish') metric spaces $X$ and compact metric spaces $K$ (represented as totally bounded complete metric spaces) theorem 5.1 yields (taking quantification over $K$ and $X$ as a kind of 'macro') the following 'applied' version: from proofs (say in $\mathrm{PA}^{\omega}+\mathrm{QF}-\mathrm{AC}+\mathrm{WKL}$ ) of theorems

$$
\text { (1) } \forall k \in \mathbb{N} \forall x \in X \forall y \in K \exists n \in \mathbb{N} A_{1}(k, x, y, n) \text {, }
$$

\footnotetext{
${ }^{6}$ In particular this is the case if we can allow the full extensionality rule resulting in a system satisfying the deduction theorem. Full extensionality may be added e.g. if the types in $\Delta$, QF-AC and $\rho$ are $\leq 1$ since then the elimination of extensionality procedure from [89] applies. One can also permit that the premise $\Delta(x, y)$ depends on the parameters $x$ and $y \leq s x$.
} 
where $A_{1}$ is, modulo the representation of $X, K$, a purely existential $\left(\Sigma_{1}^{0}\right)$-formula, one can extract bounds $\Phi \in T$ on ' $\exists n$ ' that are independent from $y \in K$ but only depend on $k$ and a representative $f_{x} \in \mathbb{N}^{\mathbb{N}}$ of the element $x \in X$, i.e.

$$
\text { (2) } \forall k \in \mathbb{N} \forall x \in X \forall y \in K \exists n \leq \Phi\left(k, f_{x}\right) A_{1}(k, x, y, n) \text {, }
$$

where $f_{x}$ is any representative of $x \in X$ (see [56]).

Remark 5.3. (Enrichment of data) As stressed by G. Kreisel since the 50's in lectures at Stanford (see also [84]) functional interpretation is a systematic tool to enrich the data of a problem in the numerical appropriate form to make an effective solution possible. E.g. a strictly positive real number $x$ gets enriched by a witness $n \in \mathbb{N}$ such that $x \geq 2^{-n}$, a Cauchy sequence gets enriched by a rate of convergence. Monotone functional interpretation (which in these simple cases produces the same enrichments as functional interpretation), moreover, enriches a continuous function, say on $[0,1]$, by a modulus of uniform continuity. Here and in many other cases it automatically creates the type of enrichments used e.g. in E. Bishop's treatment of constructive analysis ([6, 8]). Whereas Bishop himself considered the functional interpretation of implications as 'numerical implication' ([7]) it is argued in [75] that actually the monotone version is the more natural one. We will come back to this issue at the end of this paper.

Often theorems (1) result as prenex normal forms of theorems of the form

$$
\text { (3) } \forall x \in X \forall y \in K\left(F(x, y)==_{\mathbb{R}} 0 \rightarrow G(x, y)={ }_{\mathbb{R}} 0\right)
$$

for suitable $T$-definable functions $F, G: X \times K \rightarrow \mathbb{R}$. This can be rewritten as

$$
\text { (4) } \forall k \in \mathbb{N} \forall x \in X \forall y \in K \exists n \in \mathbb{N}\left(|F(x, y)| \leq_{\mathbb{R}} 2^{-n} \rightarrow|G(x, y)|<_{\mathbb{R}} 2^{-k}\right),
$$

where the matrix in (4) is logically equivalent to a $\Sigma_{1}^{0}$-formula.

Even for constructively proven theorems (3), modified realizability could not be used since it produces the empty realizer for $\exists$-free sentences such as (3). Only a technique supporting in a non-trivial way (i.e. not by unbounded search as in Kleene realizability) the (rule version of the) Markov principle at least for numbers (needed to perform the transition from (3) to (4)) such as functional interpretation is of any use here.

A particularly important class of theorems having the form (3) are uniqueness theorems

$$
\text { (5) } \forall x \in X \forall y_{1}, y_{2} \in K\left(\bigwedge_{i=1}^{2}\left(F\left(x, y_{i}\right)={ }_{\mathbb{R}} 0\right) \rightarrow y_{1}={ }_{K} y_{2}\right) .
$$

Then (2) provides a 'modulus of uniqueness' ([56]) $\Phi$ such that

$$
\forall k \in \mathbb{N} \forall x \in X \forall y_{1}, y_{2} \in K\left(\bigwedge_{i=1}^{2}\left(\left|F\left(x, y_{i}\right)\right| \leq_{\mathbb{R}} 2^{-\Phi\left(k, f_{x}\right)}\right) \rightarrow d_{K}\left(y_{1}, y_{2}\right)<\mathbb{R} 2^{-k}\right) .
$$

The crucial property of $\Phi$ is that it does not depend on $y_{1}, y_{2} \in K$ and hence can be used to compute the unique root $\widehat{y} \in K$ of $F(x, \cdot)$ in case where it exists: let $\Psi\left(n, f_{x}\right)$ be any algorithm for computing a $2^{-n}$-root in $K$, i.e.

$$
\forall n \in \mathbb{N}\left(\left|F\left(x, \Psi\left(n, f_{x}\right)\right)\right| \leq_{\mathbb{R}} 2^{-n}\right) .
$$

Then $d_{K}\left(\widehat{y}, \Psi\left(\Phi\left(k, f_{x}\right), f_{x}\right)\right)<2^{-k}$. Because of this and several other numerically significant properties, such moduli $\Phi$ feature prominently in numerical analysis under the name of 'strong unicity'. This, in particular, is the case for best approximation theory, where based on functional interpretation new results on the best Chebycheff as well as $L_{1}$-approximation of functions in $C[0,1]$ by polynomials $p \in P_{n}$ of degree $\leq n$ (for the case of Chebycheff approximation also for more general so-called Haar spaces instead of polynomials) have been obtained ([56, 57, 74]).

Whereas the difficult cases of Chebycheff and $\mathrm{L}_{1}$-approximation deal with the special spaces $C[0,1]$ and $P_{n}$ the following much simpler uniqueness result applies to a general class of spaces. 
Definition 5.4. A normed linear space $(X,\|\cdot\|)$ is called strictly convex if

$$
\forall x_{1}, x_{2} \in B\left(\left\|\frac{1}{2}\left(x_{1}+x_{2}\right)\right\|=1 \rightarrow x_{1}=x_{2}\right), \text { where } B:=\{x \in X:\|x\| \leq 1\} .
$$

Proposition 5.5. Let $(X,\|\cdot\|)$ be a strictly convex space and $C \subseteq X$ be a convex subset. Then each element $x \in X$ has at most one element $y_{b} \in C$ of best approximation in $C$, i.e. at most one element $y_{b} \in C$ such that $\left\|x-y_{b}\right\|=d:=\inf _{y \in C}\|x-y\|$.

Definition 5.6. A normed space $(X,\|\cdot\|)$ is called uniformly convex with modulus of uniform convexity $\eta:(0,2] \rightarrow(0,1]$ if

$$
\forall x_{1}, x_{2} \in B \forall \varepsilon \in(0,2]\left(\left\|\frac{1}{2}\left(x_{1}+x_{2}\right)\right\| \geq 1-\eta(\varepsilon) \rightarrow\left\|x_{1}-x_{2}\right\| \leq \varepsilon\right) .
$$

We may assume that $\eta(\varepsilon)<1$.

One easily shows that for uniformly convex $(X,\|\cdot\|)$ with modulus of uniform convexity $\eta$, convex $C \subseteq X, x \in X$ and $D \geq d$ the function

$$
\Phi(\varepsilon):=\min \left\{1, \frac{\varepsilon}{4}, \frac{\varepsilon}{4} \cdot \frac{\eta(\varepsilon /(D+1))}{1-\eta(\varepsilon /(D+1))}\right\}
$$

is a modulus of uniqueness, i.e.

$$
\forall y_{1}, y_{2} \in C \forall \varepsilon \in(0,2]\left(\bigwedge_{i=1}^{2}\left(\left\|x-y_{i}\right\| \leq d+\Phi(\varepsilon)\right) \rightarrow\left\|y_{1}-y_{2}\right\| \leq \varepsilon\right) .
$$

As an immediate corollary to this uniform uniqueness result one gets (for uniformly convex Banach spaces and closed convex $C \subseteq X$ ) the existence of a (then, of course, unique) best approximation despite of the fact that compactness arguments cannot be applied here: by the definition of $d$ there is a sequence $\left(y_{n}\right)$ in $C$ with

$$
\left\|x-y_{n}\right\| \leq d+2^{-n} .
$$

Hence $\left(y_{\Phi\left(2^{-n}\right)}\right)$ is a Cauchy sequence whose limit clearly is a best approximation.

In fact the standard well-known existence proof for best approximations in uniformly convex spaces implicitly uses this very reasoning. Note that the plain uniqueness result which follows already from the weaker assumption of strict convexity is not sufficient to conclude this.

This simple example illustrates two things:

- Despite of the fact that we did not assume $X$ to be separable or $C$ to be compact we obtained a uniform modulus of uniqueness not depending on $y_{1}, y_{2} \in C$ except for some upper bound on $\left\|x-y_{1}\right\|,\left\|x-y_{2}\right\|$ (clearly any such bound also is an upper bound on $d$ and given a bound $D$ on the latter $\left\|x-y_{i}\right\| \leq d+\Phi(\varepsilon)$ implies $\left.\left\|x-y_{i}\right\| \leq D+1\right)$. For this it was sufficient to 'uniformize' the condition of strict convexity to uniform convexity which would follow in the very special case of a compact unit ball (implying the space to be finite dimensional) but also holds in much more general contexts. In fact essentially all strictly convex spaces of interest also are uniformly convex.

- In the compact case $K$ and in contexts where all constants of the language are computable the existence of bounds which are independent from parameters in $K$ can be established using compactness arguments (even yielding an effective uniform bound): by unbounded search one gets a computable non-uniform bound. Since computable type-2 functionals are uniformly continuous when restricted to the Cantor space (effectively in further type-1 parameters) the result follows. Hence here it is the concrete (subrecursive) description of the bounds extracted from given proofs which is of interest. However, in the absence of compactness already the 
existence of a (qualitative) uniformity result can be of interest e.g. by providing an existence result which even ineffectively could not be obtained otherwise. Moreover, in the presence of abstract metric or normed spaces without any computability structure (see below) one cannot even search for an effective (non-uniform) bound. For a nontrivial new fixed point theorem which was obtained in this way by removing a compactness assumption see [11].

For concrete spaces $(X,\|\cdot\|)$, one easily can construct counterexamples to the claim that the observations just mentioned hold in general. In fact, separability (which was used in the previous metatheorem to represent $K$ via the Cantor space) now is the main obstacle as the uniform version of separability on bounded subsets is nothing else but the total boundedness of these sets and so (up to completeness) brings one back to the compact case. The situation, however, changes if a proof applies to a general class of spaces $X$ whose defining axioms have the right uniformity built-in as is the case for uniformly convex normed spaces but also many other structures including: metric spaces, hyperbolic spaces, CAT(0)-spaces, normed spaces, inner product spaces, uniformly convex hyperbolic spaces, $\mathbb{R}$-trees, $\delta$-hyperbolic spaces (see $[64,28,87]$ ). In fact general metatheorems (based on extensions of monotone functional interpretation) have been proved which guarantee the extractability of effective uniform bounds which only depend on certain local upper bounds on metric distances of parameters in $X$, sequences in $X$ and functions $f: X \rightarrow X$. Since the main interest here is in new qualitative uniformity results we will work in extensions of the system of classical analysis $\mathcal{A}^{\omega}:=\mathrm{PA}^{\omega}+\mathrm{QF}-\mathrm{AC}+\mathrm{DC}$ as treated by Spector and Howard using bar recursion $([102,49])$. Although the latter has a complexity too vast to be of any numerical use, it does yield effective uniform bounds when combined with a novel majorization relation (see below). Moreover, for concrete proofs usually only small fragments of the systems will be used so that the bounds actually extracted often do have numerical value (see [67] for a survey).

In order to formalize proofs dealing with abstract classes of structures $X$ such as general metric or normed spaces we add such structures as kind of atoms ('Urelemente') to the system $\mathcal{A}^{\omega}$ by extending the set of types $\mathbf{T}$ to a new base type $X$ (ranging over elements from $X$ ) over which we form the set of all finite types (in fact one can add several such structures simultaneously but we treat here only the case of one structure). Then by adding appropriate new constants and axioms one axiomatizes the class of structures at hand. In such a framework one can formalize proofs of theorems which hold for all structures in the class being axiomatized (treated as parameters) as long as we only consider theorems that do not quantify over the class of structures.

For proofs in intuitionistic mathematics this approach has already clearly been anticipated in Gödel's early 1941 presentation of functional interpretation where he writes

'more generally, if you apply intuitionistic logic in any branch of mathematics, you can reduce it to a finitistic system of this kind under the sole hypothesis that the primitive functions and primitive recursive relations of this branch of mathematics are calculable, respectively, decidable. ... This finitistic system to which intuitionistic logic, applied in the branch of mathematics under consideration, can be reduced is always obtained by introducing functions of higher types analogous to these, with the only difference that the individuals upon which the hierarchy of functions is built up are no longer the integers but the primitive objects of the branch of mathematics under consideration.' Gödel ([36], pp.195-196).

Definition 5.7. The set $\mathbf{T}^{X}$ of all finite types over the two ground types 0 and $X$ is defined by

$$
\text { (i) } 0, X \in \mathbf{T}^{X} \text {, (ii) } \rho, \tau \in \mathbf{T}^{X} \Rightarrow(\rho \rightarrow \tau) \in \mathbf{T}^{X} \text {. }
$$

A type is called small if it is of degree $\leq 1$ (i.e. $0 \rightarrow \ldots \rightarrow 0 \rightarrow 0$ ) or the form $\rho_{1} \rightarrow \ldots \rightarrow \rho_{k} \rightarrow X$, where the $\rho_{i}$ 's are 0 or $X$.

The theories $\mathcal{A}^{\omega}[X, d]_{-b}$ and $\mathcal{A}^{\omega}[X, d, W]_{-b}$ result ${ }^{7}$ by extending $\mathcal{A}^{\omega}$ to all types in $\mathbf{T}^{X}$ and adding

\footnotetext{
${ }^{7}$ The index ' $-b$ ' indicates that in contrast to the corresponding theories in [64] we (following [28]) do not require the metric space to be bounded.
} 
axioms for an abstract metric (in the case of $\mathcal{A}^{\omega}[X, d]_{-b}$ ) resp. hyperbolic (in the case of $\mathcal{A}^{\omega}[X, d, W]_{-b}$ ) space. $\mathcal{A}^{\omega}[X, d, W, \mathrm{CAT}(0)]_{-b}$ is the extension by an abstract $\mathrm{CAT}(0)$-space. Analogously, one has theories $\mathcal{A}^{\omega}[X,\|\cdot\|]$ with an abstract non-trivial real normed space added (as well as further extensions $\mathcal{A}^{\omega}[X,\|\cdot\|, C]$ resp. $\mathcal{A}^{\omega}[X,\|\cdot\|, C]_{-b}$ with bounded resp. general convex subsets $C \subseteq X$ which we will, however, due to lack of space not treat here). Our theories also contain a constant $0_{X}$ of type $X$ which in the normed case represents the zero vector and in the other cases stands for an arbitrary element of the metric space. For details on all this see [64, 28].

Equality $=_{X}$ for the new type $X$ is a defined notion

$$
x={ }_{X} y: \equiv\left(d_{X}(x, y)={ }_{\mathbb{R}} 0_{\mathbb{R}}\right)
$$

and so we still only have decidable prime formulas $s={ }_{0} t$. Since we have to work in the weakly extensional setting of Spector's quantifier-free extensionality we do not have

$$
x={ }_{X} y \rightarrow f^{X \rightarrow X}(x)={ }_{X} f(y)
$$

but only from a proof of $A_{0} \rightarrow s={ }_{X} t$ can infer that $A_{0} \rightarrow f(s)={ }_{X} f(t)$ ( $A_{0}$ quantifier-free). This is of crucial importance for our metatheorems to hold. Fortunately, we can in most cases prove the extensionality of $f$ for those functions we consider, e.g. for nonexpansive functions, so that this only causes some need for extra care in few cases (for an extensive discussion of this point see [64]).

Definition 5.8. For $\rho \in \mathbf{T}^{X}$ we define $\widehat{\rho} \in \mathbf{T}$ inductively as follows

$$
\widehat{0}:=0, \widehat{X}:=0,(\widehat{\rho \rightarrow \tau}):=(\widehat{\rho} \rightarrow \widehat{\tau}),
$$

i.e. $\widehat{\rho}$ is the result of replacing all occurrences of the type $X$ in $\rho$ by the type 0 .

We now introduce an extension of the majorization relation to objects with types $\rho \in \mathbf{T}^{X}$ where, however, the majorants always have types $\widehat{\rho} \in \mathbf{T}$. This relation is parametrized by an arbitrary reference point $a \in X$.

Definition 5.9 ([28]). We define a ternary majorization relation $\gtrsim_{\rho}^{a}$ between objects $x, y$ and $a$ of type $\widehat{\rho}, \rho$ and $X$, respectively, by induction on (the depth of) $\rho$ as follows: ${ }^{8}$

- $x^{0} \gtrsim_{0}^{a} y^{0}: \equiv x \geq y$,

- $x^{0} \gtrsim_{X}^{a} y^{X}: \equiv(x)_{\mathbb{R}} \geq_{\mathbb{R}} d_{X}(y, a)$,

- $x \gtrsim_{\rho \rightarrow \tau}^{a} y: \equiv \forall z^{\prime}, z\left(z^{\prime} \quad \gtrsim_{\rho}^{a} \quad z \rightarrow x\left(z^{\prime}\right) \gtrsim_{\tau}^{a} y(z)\right) \wedge \forall z^{\prime}, z\left(z^{\prime} \gtrsim_{\widehat{\rho}}^{a} \quad z \rightarrow x\left(z^{\prime}\right) \gtrsim_{\tilde{\tau}}^{a} x(z)\right)$.

For normed linear spaces we choose $a=0_{X}$.

Definition 5.10. A formula $F$ in $\mathcal{L}\left(\mathcal{A}^{\omega}[X, \ldots]_{-b}\right)$ is called $\forall$-formula (resp. $\exists$-formula) if it has the form $F \equiv \forall \underline{a} \underline{\underline{\sigma}} F_{q f}(\underline{a})$ (resp. $F \equiv \exists \underline{a} \underline{\underline{\sigma}} F_{q f}(\underline{a})$ ) where $F_{q f}$ does not contain any quantifier and the types in $\underline{\sigma}$ are small.

Theorem 5.11 ([28]). $\quad$ 1) Let $\rho$ be a small type and let $A_{\forall}(x, u)$, resp. $B_{\exists}(x, v)$, be $\forall$ - and $\exists$ formulas that contain only $x, u$ free, resp. $x, v$ free. Assume that the constant $0_{X}$ does not occur in $A_{\forall}, B_{\exists}$ and that

$$
\mathcal{A}^{\omega}[X, d]_{-b} \vdash \forall x^{\rho}\left(\forall u^{0} A_{\forall}(x, u) \rightarrow \exists v^{0} B_{\exists}(x, v)\right) .
$$

Then one can extract a computable functional ${ }^{9} \Phi: S_{\widehat{\rho}} \rightarrow \mathbb{N}$ such that the following holds in all nonempty metric spaces $(X, d):$ for all $x \in S_{\rho}, x^{*} \in S_{\widehat{\rho}}$ if there exists an $a \in X$ s.t. $x^{*} \gtrsim^{a} x$ then

$$
\forall u \leq \Phi\left(x^{*}\right) A_{\forall}(x, u) \rightarrow \exists v \leq \Phi\left(x^{*}\right) B_{\exists}(x, v) .
$$

\footnotetext{
${ }^{8}$ Here $(x)_{\mathbb{R}}$ refers to the embedding of $\mathbb{N}$ into $\mathbb{R}$ in the sense of our representation of $\mathbb{R}$.

${ }^{9}$ Note that for small types $\rho$ the type $\widehat{\rho}$ is of degree 1 . So $\Phi$ essentially is a type- 2 functional $: \mathbb{N}^{\mathbb{N}} \rightarrow \mathbb{N}$.
} 
2) The theorem also holds for nonempty hyperbolic spaces $\mathcal{A}^{\omega}[X, d, W]_{-b}$, $(X, d, W)$ and for $\mathcal{A}^{\omega}[X, d, W, \mathrm{CAT}(0)]_{-b}$, where $(X, d)$ is a $\mathrm{CAT}(0)$-space.

3) The theorem also holds for non-trivial real normed spaces $\mathcal{A}^{\omega}[X,\|\cdot\|],(X,\|\cdot\|)$, where then ' $a$ ' has to be interpreted by the zero vector in $(X,\|\cdot\|)$ and $0_{X}$ is allowed to occur in $A_{\forall}, B_{\exists}$. Instead of single variables $x, u, v$ and single premises $\forall u A_{\forall}(x, u)$ we may have tuples of variables and finite conjunctions of premises. In the case of a tuple $\underline{x}$ we then have to require that we have a tuple $\underline{x}^{*}$ of a-majorants for a common $a \in X$ for all the components of the tuple $\underline{x}$.

Remark 5.12. From the proof of theorem 5.11 it follows that the theorem also holds with additional purely universal axioms and majorizable constants of sufficiently small types added where then the bound depends on those majorants. Based on this, the theorem above has been adapted to other structures such as uniformly convex normed spaces or inner product spaces ([28]) as well as to uniformly convex hyperbolic spaces, $\delta$-hyperbolic spaces (in the sense of M. Gromov) and $\mathbb{R}$-trees in the sense of Tits (see [87]).

Since the bound $\Phi$ operates on objects of degree $\leq 1$, i.e. natural numbers or $n$-ary number theoretic functions rather than $x \in X$ or $f: X \rightarrow X$ the usual type-2 computability theory as well as wellknown subrecursive classes of such functionals apply here irrespectively of whether the metric or normed spaces to which the bounds are applied come with any notion of computability or not.

The proof of theorem 5.11 provides an algorithm based on (monotone) functional interpretation for the extraction of $\Phi$.

In the concrete applications, theorem 5.11 is used via various applied corollaries of which we give an example now:

Definition 5.13. Let $(X, d)$ be a metric space. A mapping $f: X \rightarrow X$ is called nonexpansive if

$$
\forall x, y \in X(d(f(x), f(y)) \leq d(x, y)) .
$$

Corollary 5.14 ([28]). Let $A_{\exists}$ be an $\exists$-formula and $P, K$ Polish resp. compact metric spaces in standard representation by $\mathcal{A}^{\omega}$-definable terms (see [56] for a precise definition). If $\mathcal{A}^{\omega}[X, d, W]_{-b}$ proves a sentence

$$
\forall x \in P \forall y \in K \forall z^{X}, \tilde{z}^{X}, f^{X \rightarrow X}\left(f \text { nonexpansive } \rightarrow \exists v^{\mathbb{N}} A_{\exists}\right)
$$

then one can extract a computable functional $\Phi\left(g_{x}, b\right)$ s.t. for all $x \in P, g_{x} \in \mathbb{N}^{\mathbb{N}}$ representative of $x, b \in \mathbb{N}$

$$
\forall y \in K \forall z, \tilde{z} \in X \forall f: X \rightarrow X\left(f \text { n.e. } \wedge d(z, f(z)), d(z, \tilde{z}) \leq b \rightarrow \exists v \leq \Phi\left(g_{x}, b\right) A_{\exists}\right)
$$

holds in any nonempty hyperbolic space $(X, d, W)$.

Proof (sketch): The fact that $P, K$ have a standard representation by $\mathcal{A}^{\omega}$-terms essentially means that $\forall$-quantification over $P$ resp. $K$ can be expressed as quantification $\forall x^{1}$ resp. $\forall y \leq_{1} N$ where $N$ is a fixed simple (primitive recursive) function depending on $K$. Here the number theoretic functions encode Cauchy sequences (with fixed rate of convergence) of elements from the countable dense subset of $P$ resp. $K$ on which the standard representations are based. We now apply theorem 5.11 with $a:=z$. For this we have to construct $\gtrsim^{z}$-majorants for $x^{1}, y^{1}, z^{X}, \tilde{z}^{X}$ and $f^{X \rightarrow X}$ :

$$
x^{*}:=x^{M}:=\lambda n . \max \{x(i): i \leq n\}, y^{*}:=N^{M}, z^{*}:=0^{0}, \tilde{z}^{*}:=b, f^{*}:=\lambda n^{0} . n+b .
$$

For $f^{*}$ we use that

$$
d(x, z) \leq n \rightarrow d(f(x), z) \leq d(f(x), f(z))+d(f(z), z) \leq d(x, z)+d(f(z), z) \leq n+b .
$$

Note that the majorants only depend on $x, b$. 
Remark 5.15. The above corollary holds similarly for the case of normed and inner product spaces (as well as their completions, see [69]) but - due to the fact that then ' $a$ ' must be fixed as the zero vector $0_{X}$ - one has to add the assumption $\|z\| \leq b$ in the conclusion.

We conclude this section with one concrete application of corollary 5.14 : Let $(X, d, W)$ be a hyperbolic space, $f: X \rightarrow X$ nonexpansive and $\left(\lambda_{n}\right)$ be a sequence in $[0,1]$ which is bounded away from 1 and divergent in sum. Let $\left(x_{n}\right)$ be the so-called Krasnoselski-Mann iteration starting from $x_{0}:=x \in X:$

$$
x_{n+1}:=\left(1-\lambda_{n}\right) x_{n} \oplus \lambda_{n} f\left(x_{n}\right), \text { where }(1-\lambda) x \oplus \lambda y \text { denotes } W(x, y, \lambda) .
$$

\section{Theorem 5.16 (Borwein-Reich-Shafrir [9]).}

$$
\forall x \in X\left(d\left(x_{n}, f\left(x_{n}\right)\right) \stackrel{n \rightarrow \infty}{\rightarrow} r(f):=\inf _{y \in X} d(y, f(y))\right)
$$

As shown in [28], corollary 5.14 implies that there exists a computable function $\Phi: \mathbb{N}^{\mathbb{N}} \times \mathbb{N}^{3} \rightarrow \mathbb{N}$ such that (using that $\left(d\left(x_{n}, f\left(x_{n}\right)\right)\right)_{n}$ is nonincreasing) :

For all hyperbolic spaces $X$, all nonexpansive functions $f: X \rightarrow X$, all $\left(\lambda_{n}\right)$ in $[0,1], k \in \mathbb{N}$, $\alpha: \mathbb{N} \rightarrow \mathbb{N}$, with $\forall n\left(\lambda_{n} \leq 1-\frac{1}{k} \wedge n \leq \sum_{i=0}^{\alpha(n)} \lambda_{i}\right)$ and all $x, x^{*} \in X, b \in \mathbb{N}$ with $b \geq d\left(x, x^{*}\right), d(x, f(x)):$

$$
\forall l \in \mathbb{N} \forall n \geq \Phi(\alpha, k, b, l)\left(d\left(x_{n}, f\left(x_{n}\right)\right)<d\left(x^{*}, f\left(x^{*}\right)\right)+2^{-l}\right) .
$$

Such a $\Phi$ has been extracted in [71] (for the normed case already in [62]) from the original ineffective proof in [9]:

$$
\begin{aligned}
& \Phi(\alpha, k, b, l):=\widehat{\alpha}(\lceil 2 b \cdot \exp (k(M+1))\rceil-1, M), \text { where } \\
& M:=(1+2 b) \cdot 2^{l}, \widehat{\alpha}(0, n):=\tilde{\alpha}(0, n), \widehat{\alpha}(i+1, n):=\tilde{\alpha}(\widehat{\alpha}(i, n), n), \text { with } \\
& \tilde{\alpha}(i, n):=i+\alpha^{+}(i, n), \text { where } \alpha^{+}(i, n):=\max _{j \leq i}[\alpha(n+j)-j+1] .
\end{aligned}
$$

For various applications of this result (also new qualitative results which only use the uniformity of the bound but not its numerical value) see [71] and [72]. For uniformly convex hyperbolic spaces often special arguments yielding better bounds apply. This, in particular, covers the important class of CAT(0)-spaces in the sense of Gromov and in many cases provides even quadratic bounds. Here is one example: Let $f: C \rightarrow C$ be a selfmapping of a convex subset $C$ of some $\mathrm{CAT}(0)$-space $(X, d)$. $f$ is called asymptotically nonexpansive if for some sequence $\left(k_{n}\right)$ in $[0, \infty)$ with $\lim _{n \rightarrow \infty} k_{n}=0$ one has

$$
d\left(f^{n} x, f^{n} y\right) \leq\left(1+k_{n}\right) d(x, y), \quad \forall n \in \mathbb{N}, \forall x, y \in C .
$$

In this case, the Krasnoselski-Mann iteration of $f$ starting from $x \in C$ is defined by

$$
x_{0}:=x, x_{n+1}:=\left(1-\lambda_{n}\right) x_{n} \oplus \lambda_{n} f^{n}\left(x_{n}\right) .
$$

Based on a suitable variant of corollary 5.14, the following quadratic (in $\varepsilon$ ) bound has been extracted recently:

Theorem 5.17 ([73]). Let $(X, d)$ be a CAT(0)-space, $C$ be a nonempty convex bounded subset of $X$ whose diameter is bounded by $d_{C}$ and let $f: C \rightarrow C$ be asymptotically nonexpansive with sequence $\left(k_{n}\right)$.

Assume that $K \geq 0$ is such that $\sum_{n=0}^{\infty} k_{n} \leq K$ and that $L \in \mathbb{N}, L \geq 2$ is such that $\frac{1}{L} \leq \lambda_{n} \leq 1-\frac{1}{L}$ for all $n \in \mathbb{N}$.

Then the following holds for all $x \in C$ :

$$
\forall \varepsilon \in(0,1] \exists n \leq \Phi\left(K, L, d_{C}, \varepsilon\right)\left(d\left(x_{n}, f\left(x_{n}\right)\right)<\varepsilon\right),
$$


where

$$
\begin{aligned}
& \Phi\left(K, L, d_{C}, \varepsilon\right):=2 M, \\
& M:=\left\lceil\frac{1}{\varepsilon^{2}} \cdot 24 L^{2}\left(5 K D+D+\frac{11}{2}\right)(h(K))^{3}((1+K) D+1)^{2}\right\rceil, \\
& D:=e^{K}\left(d_{C}+2\right), \quad h(K):=2\left(1+(1+K)^{2}(2+K)\right) .
\end{aligned}
$$

As mentioned above, the extraction technique of monotone functional interpretation underlying the proof of corollary 5.14 (in the form for Hilbert spaces) has recently been used in [4] to extract a uniform bound on the Herbrand normal form of the von Neumann Mean Ergodic Theorem (see also $[107])$.

\section{Concluding remarks}

Foundational reductions revisited: As discussed above, Gödel's aim in developing functional interpretation (and negative translation) was to give a consistency proof for PA by reducing it to $T$ which Gödel considered as a legitimate extension of strict finitism in the narrow sense. The foundational relevance of this reduction (and other consistency proofs) remains debatable (see A.S. Troelstra's introductory remarks to [37] in [40]). The shift of emphasis towards applications in mathematics deviates from this original motivation and replaces the issue of foundational reductions by concrete mathematical applications. However, there is one aspect of the original preoccupation with consistency proofs that has shown up again in the course of this applied reorientation: monotone functional interpretation can be used to prove a useful elimination result for a classically false strong uniform boundedness principle $\exists-\mathrm{UB}^{X}$ ([66]) over e.g. $\mathcal{A}^{\omega}[X, d, W]$ (i.e. $\mathcal{A}^{\omega}[X, d, W]_{-b}$ plus an axiom stating the boundedness of $(X, d)$ ). This principle allows one (among many other things) to prove (over $\mathcal{A}^{\omega}[X, d, W]$ ) that every nonexpansive mapping $f: X \rightarrow X$ has a fixed point which is known to be false already for bounded closed convex subsets of Banach spaces such as $c_{0}$. Nevertheless, for a large class of sentences $A$ provable using $\exists-\mathrm{UB}^{X}$ (including so-called asymptotic regularity statements) one can show that they are classically correct (see [66] and [63, 70] for concrete instances of this). Since in metric fixed point theory many proofs of asymptotic regularity exist which use as an assumption that $f$ has fixed points this can (and has been) applied for

- removing the need of complicated fixed point theorems used to cancel this assumption (resulting in elementary proofs) and

- at the same time dropping assumptions only needed to have these fixed point theorems available (thereby generalizing proofs).

Enrichment of data revisited: Whereas monotone functional interpretation over the original types $\mathbf{T}$ over $\mathbb{N}$ create (irrespectively of whether negative translation is used first or not) constructive enrichments of data which classically are redundant (for statements involving continuous functions only) our extension to the types for abstract classes of spaces based on $\gtrsim^{a}$ creates enrichments which even classically are a proper strengthening of the original assumptions (due to the lack of compactness). Using the uniform boundedness principle $\exists-\mathrm{UB}^{X}$ just mentioned these enrichments become provable. E.g. we translate for bounded metric or hyperbolic spaces

- separability $\Rightarrow$ total boundedness (with modulus)

- extensionality $\Rightarrow$ uniform continuity (with modulus) ${ }^{10}$

- pointwise monotone convergence $\Rightarrow$ uniform convergence (with modulus)

- existence of approximate solutions $\Rightarrow$ existence of solutions

\footnotetext{
${ }^{10}$ This illustrates the need to be restrictive w.r.t. extensionality.
} 
- strict convexity $\Rightarrow$ uniform convexity (with modulus) in the hyperbolic or normed case

- contractive functions (Edelstein) $\Rightarrow$ uniformly contractive functions (with modulus, Rakotch)

- uniqueness $\Rightarrow$ uniform uniqueness (with modulus).

What essentially is shown by theorems such as theorem 5.11 is that provided we restrict us to input data having these uniformity features then this uniformity prevails throughout even prima facie highly ineffective proofs formalizable in $\mathcal{A}^{\omega}[X, d, W]$ and yields an effective uniform version of the theorem in question. In many concrete applications even ineffectively the uniform version was not known before.

Acknowledgement: The author is grateful to Professor G. Kreisel for numerous comments on an earlier version of this article.

\section{References}

[1] Ackermann, W., Zur Widerspruchsfreiheit der reinen Zahlentheorie. Math. Ann. 117, pp. 162194 (1940).

[2] Artemov, S., Explicit provability and constructive semantics. Bull. Symbolic Logic 7, pp. 1-36 (2001).

[3] Avigad, J., Feferman, S., Gödel's functional ('Dialectica') interpretation. In: [16], pp. 337-405 (1998).

[4] Avigad, J., Gerhardy, P., Towsner, H., Local stability of ergodic averages. arXiv:0706.1512v1 [math.DS] (2007).

[5] Bezem, M., Strongly majorizable functionals of finite type: a model for bar recursion containing discontinuous functionals. J. Symbolic Logic 50 pp. 652-660 (1985).

[6] Bishop, E., Foundations of Constructive Analysis. New York, McGraw-Hill, 1967.

[7] Bishop, E., Mathematics as a numerical language. In: Kino, Myhill, Vesley (eds.), Intuitionism and Proof Theory, pp. 53-71, North-Holland, Amsterdam (1970).

[8] Bishop, E., Bridges, D., Constructive Analysis. Springer-Verlag, Berlin Heidelber New York Tokyo, xii+477 pp., 1985.

[9] Borwein, J., Reich, S., Shafrir, I., Krasnoselski-Mann iterations in normed spaces. Canad. Math. Bull. 35, pp. 21-28 (1992).

[10] Briseid, E.M., Proof mining applied to fixed point theorems for mappings of contractive type. Master Thesis, Oslo, 70pp., 2005.

[11] Briseid, E.M., Fixed points of generalized contractive mappings. To appear in: J. of Nonlinear and Convex Analysis.

[12] Briseid, E.M., A rate of convergence for asymptotic contractions. J. Math. Anal. Appl. 330, pp. 364-376 (2007).

[13] Briseid, E.M., Some results on Kirk's asymptotic contractions. Fixed Point Theory 8, No.1, pp. 17-27 (2007).

[14] Briseid, E.M., Proof mining and mappings of contractive type. In preparation. 
[15] Burr, W., Functional interpretation of Aczel's constructive set theory. Ann. Pure Applied Logic 104, pp. 31-73 (2000).

[16] Buss, S.R. (editor), Handbook of Proof Theory. Studies in Logic and the Foundations of Mathematics Vol 137, Elsevier, vii+811 pp. (1998).

[17] Cook, S., Urquhart, A., Functional interpretations of feasibly constructive arithmetic. Ann. Pure Applied Logic 63, pp. 103-200 (1993).

[18] Coquand, T., Hofmann, M., A new method for establishing conservativity of classical systems over their intuitionistic version. Lambda-calculus and logic. Math. Structures Comput. Sci. 9, pp. 323-333 (1999).

[19] Diller, J., Nahm, W., Eine Variante zur Dialectica-Interpretation der Heyting-Arithmetik endlicher Typen. Arch. Math. Logik Grundlagenforsch. 16, pp. 49-66 (1974).

[20] Dragalin, A.G., New kinds of realizability and the Markov rule. Dokl. Akad. Nauk. SSSR 251, pp. 534-537 (1980) (Russian). English translation: Soviet Math. Dokl. 21, pp. 461-464 (1980).

[21] Feferman, S., Kreisel's 'Unwinding Program'. In: P. Odifreddi (ed.), Kreiseliana: about and around Georg Kreisel, A.K. Peters, Wellesley Massachusetts, pp. 247-273 (1996).

[22] Ferreira, F., Oliva, P., Bounded functional interpretation. Ann. Pure Appl. Logic 135, pp. 73-112 (2005).

[23] Friedman, H., Classical and intuitionistically provably recursive functions. In: Müller,G.H., Scott, D.S. (eds.), Higher Set Theory, pp. 21-27. Springer LNM 669 (1978).

[24] Friedrich, W., Gödelsche Funktionalinterpretation für eine Erweiterung der klassischen Analysis. Zeitschr. f. math. Logik und Grundl. d. Math. 31, pp. 3-29 (1985).

[25] Gerhardy, P., A quantitative version of Kirk's fixed point theorem for asymptotic contractions. J. Math. Anal. Appl. 316, pp. 339-345 (2006).

[26] Gerhardy, P., Kohlenbach, U., Extracting Herbrand disjunctions by functional interpretation. Arch. Math. Logic 44, pp. 633-644 (2005).

[27] Gerhardy, P., Kohlenbach, U., Strongly uniform bounds from semi-constructive proofs. Ann. Pure Appl. Logic 141, 89-107 (2006).

[28] Gerhardy, P., Kohlenbach, U., General logical metatheorems for functional analysis. Trans. Amer. Math. Soc. 360, 2615-2660 (2008).

[29] Girard, J.-Y., Une extension de l'interpretation de Gödel à l'analyse, et son application à l' élimination des coupures dans l'analyse et dans la théorie des types. In: Fenstad, J.E. (ed.), Proc. of the Second Scandinavian Logic Symposium, North-Holland, Amsterdam, pp. 63-92 (1971).

[30] Girard, J.-Y., Proof Theory and Logical Complexity Vol.I. Studies in Proof Theory. Bibliopolis (Napoli) and Elsevier Science Publishers (Amsterdam) 1987.

[31] Glivenko, V.I., Sur quelques points de la logique de M. Brouwer. Bull. Soc. Math. Belg. 15, pp. 183-188 (1929).

[32] Gödel, K., Zur intuitionistischen Arithmetik und Zahlentheorie. Ergebnisse eines Mathematischen Kolloquiums, vol. 4 pp. 34-38 (1933). Reprinted together with an English translation in: [39]. 
[33] Gödel, K., Eine Interpretation des intuitionistischen Aussagenkalküls. Ergebnisse eines Mathematischen Kolloquiums, vol. 4 pp. 39-40 (1933). Reprinted together with an English translation in: [39].

[34] Gödel, K., Vortrag bei Zilsel (1938). First published together with an English translation in: [41], pp. 86-113 (1995).

[35] Gödel, K., The consistency of the axiom of choice and of the generalized continuum hypothesis with the axioms of set theory. Annals of mathematics studies, vol. 3, Princeton University Press (1940).

[36] Gödel, K., In what sense is intuitionistic logic constructive. Lecture at Yale (1941). First published in: [41], pp. 189-200 (1995).

[37] Gödel, K., Über eine bisher noch nicht benützte Erweiterung des finiten Standpunktes. Dialectica 12, pp. 280-287 (1958). Reprinted together with an English translation in: [40].

[38] Gödel, K., On an extension of finitary mathematics which has not yet been used (1972). First published in: [40], pp. 271-280 (1990).

[39] Gödel, K., Collected Work, Vol.1, S. Feferman et al. eds. OUP. New York 1986.

[40] Gödel, K., Collected Work, Vol.2, S. Feferman et al. eds. OUP. New York 1990.

[41] Gödel, K., Collected Work, Vol.3, S. Feferman et al. eds. OUP. New York 1995.

[42] Gödel, K., Collected Work, Vol.4, S. Feferman et al. eds. OUP. New York 2003.

[43] Gödel, K., Collected Work, Vol.5, S. Feferman et al. eds. OUP. New York 2003.

[44] Herbrand, J., Logic Writings. Edited by W.D. Goldfarb. D. Reidel Publishing Company, Dordrecht-Holland (1971).

[45] Hernest, M.-D., Light functional interpretation. An optimization of Gödel's technique twoards the extraction of (more) efficient programs from (classical) proofs. In: Ong, L. (ed.), CSL 2005. Springer LNCS 3634, pp. 477-492 (2005).

[46] Hernest, M.-D., The MinLog proof-system for Dialectica program-extraction. Free software available at http://www.brics.dk/ danher/MinLogForDialectica.

[47] Hernest, M.-D., Kohlenbach, U., A complexity analysis of functional interpretations. Theoretical Computer Science 338, pp. 200-246 (2005).

[48] Hilbert, D., Über das Unendliche. Math. Ann. 95, pp. 161-190 (1926).

[49] Howard, W.A., Functional interpretation of bar induction by bar recursion. Compositio Mathematica 20, pp. 107-124 (1968).

[50] Howard, W.A., Hereditarily majorizable functionals of finite type. In: Troelstra (ed.), Metamathematical investigation of intuitionistic arithmetic and analysis, pp. 454-461. Springer LNM 344 (1973).

[51] Howard, W.A., Ordinal analysis of simple cases of bar recursion. J. Symbolic Logic 46, pp. 17-30 (1981).

[52] Jørgensen, K.F., Finite Type Arithmetic: computable existence analysed by modified realizability and functional interpretation. Master Thesis, University of Roskilde, viii+121 pages, 2001. 
[53] Jørgensen, K.F., Functional interpretation and the existence property. Math. Logic Quart. 50, pp. 573-576 (2004).

[54] Kleene, S.C., On the interpretation of intuitionistic number theory. J. Symbolic Logic 10, pp. 109-124 (1945).

[55] Kohlenbach, U., Effective bounds from ineffective proofs in analysis: an application of functional interpretation and majorization. J. Symbolic Logic 57, pp. 1239-1273 (1992).

[56] Kohlenbach, U., Effective moduli from ineffective uniqueness proofs. An unwinding of de La Vallée Poussin's proof for Chebycheff approximation. Ann. Pure Appl. Logic 64, pp. 27-94 (1993).

[57] Kohlenbach, U., New effective moduli of uniqueness and uniform a-priori estimates for constants of strong unicity by logical analysis of known proofs in best approximation theory. Numer. Funct. Anal. and Optimiz. 14, pp. 581-606 (1993).

[58] Kohlenbach, U., Analysing proofs in analysis. In: W. Hodges, M. Hyland, C. Steinhorn, J. Truss, editors, Logic: from Foundations to Applications. European Logic Colloquium (Keele, 1993), pp. 225-260, Oxford University Press (1996).

[59] Kohlenbach, U., Mathematically strong subsystems of analysis with low rate of growth of provably recursive functionals. Arch. Math. Logic 36, pp. 31-71 (1996).

[60] Kohlenbach, U., On the no-counterexample interpretation. J. Symbolic Logic 64, pp. 1491-1511 (1999).

[61] Kohlenbach, U., On the computational content of the Krasnoselski and Ishikawa fixed point theorems. In: Proceedings of the Fourth Workshop on Computability and Complexity in Analysis, J. Blanck, V. Brattka, P. Hertling (eds.), Springer LNCS 2064, pp. 119-145 (2001).

[62] Kohlenbach, U., A quantitative version of a theorem due to Borwein-Reich-Shafrir. Numer. Funct. Anal. and Optimiz. 22, pp. 641-656 (2001).

[63] Kohlenbach, U., Uniform asymptotic regularity for Mann iterates. J. Math. Anal. Appl. 279, pp. 531-544 (2003).

[64] Kohlenbach, U., Some logical metatheorems with applications in functional analysis. Trans. Amer. Math. Soc. 357, no. 1, pp. 89-128 (2005) [Some minor errata are corrected at the end of [27]].

[65] Kohlenbach, U., Some computational aspects of metric fixed point theory. Nonlinear Analysis 61, pp. 823-837 (2005).

[66] Kohlenbach, U., A logical uniform boundedness principle for abstract metric and hyperbolic spaces. Electronic Notes in Theoretical Computer Science (Proc. WoLLIC 2006), 165, pp. 8193 (2006).

[67] Kohlenbach, U., Effective uniform bounds from proofs in abstract functional analysis. To appear in: Cooper, B., Loewe, B., Sorbi, A. (eds.), New Computational Paradigms: Changing Conceptions of What is Computable'. Springer Publisher, pp. 223-258 (2008).

[68] Kohlenbach, U., Proof Interpretations and the Computational Content of Proofs in Mathematics. Bulletin EATCS 93, pp., 143-173 (2007).

[69] Kohlenbach, U., Applied Proof Theory: Proof Interpretations and their Use in Mathematics. Springer Monograph in Mathematics, ca. xix+532pp. (2008). 
[70] Kohlenbach, U., Lambov, B., Bounds on iterations of asymptotically quasi-nonexpansive mappings. In: G-Falset, J., L-Fuster, E., Sims, B. (eds.), Proc. International Conference on Fixed Point Theory, Valencia 2003, pp. 143-172, Yokohama Press 2004.

[71] Kohlenbach, U., Leustean, L., Mann iterates of directionally nonexpansive mappings in hyperbolic spaces. Abstract and Applied Analysis, vol. 2003, no.8, pp. 449-477 (2003).

[72] Kohlenbach, U., Leuştean, L., The approximate fixed point property in product spaces. Nonlinear Analysis 66, pp. 806-818 (2007).

[73] Kohlenbach, U., Leuștean, L., Asymptotically nonexpansive mappings in uniformly convex hyperbolic spaces. arXiv:0707.1626 [math.LO] (2007).

[74] Kohlenbach, U., Oliva, P., Proof mining in $L_{1}$-approximation. Ann. Pure Appl. Logic 121, pp. $1-38$ (2003).

[75] Kohlenbach, U., Oliva, P., Proof mining: a systematic way of analysing proofs in mathematics. Proc. Steklov Inst. Math. 242, pp. 136-164 (2003).

[76] Kreisel, G., On the interpretation of non-finitist proofs, part I. J. Symbolic Logic 16, pp.241-267 (1951).

[77] Kreisel, G., On the interpretation of non-finitist proofs, part II: Interpretation of number theory, applications. J. Symbolic Logic 17, pp. 43-58 (1952).

[78] Kreisel, G., Interpretation of analysis by means of constructive functionals of finite types. In: Heyting, A. (ed.), Constructivity in Mathematics, North-Holland, Amsterdam, pp. 101-128 (1959).

[79] Kreisel, G., On weak completeness of intuitionistic predicate logic. J. Symbolic Logic 27, pp.139158 (1962).

[80] Kreisel, G., Foundations of intuitionistic logic. In: Proc. Logic Methodology and Philosophy of Science, Nagel, E., Suppes, P., Tarski, A. (eds.). Stanford University Press (Stanford), pp. 198-210 (1962).

[81] Kreisel, G., Review of [99]. In: Mathematical Reviews 1967. 37 \# 1224.

[82] Kreisel, G., Logical aspects of computation: contributions and distractions. In: Odifreddi, P. (ed.), Logic and Computer Science, Academic Press, London, pp. 205-278 (1990).

[83] Kreisel, G., Review of [40]. J. Symbolic Logic 56, pp. 1085-1089 (1991).

[84] Kreisel, G., Macintyre, A., Constructive logic versus algebraization I. In: Troelstra, A.S., van Dalen, D. (eds.), Proc. L.E.J. Brouwer Centenary Symposium (Noordwijkerhout 1981), NorthHolland (Amsterdam), pp. 217-260 (1982).

[85] Krivine, J.-L., Opérateurs de mise en mémoire et traduction de Gödel. Arch. Math. Logic 30, pp. 241-267 (1990).

[86] Kuroda, S., Intuitionistische Untersuchungen der formalistischen Logik. Nagoya Math. 3, pp. $35-47$ (1951).

[87] Leustean, L., Proof mining in $\mathbb{R}$-trees and hyperbolic spaces. Electronic Notes in Theoretical Computer Science (Proc. WoLLIC 2006), 165, pp. 95-106 (2006).

[88] Leustean, L., A quadratic rate of asymptotic regularity for CAT(0)-spaces. J. Math. Anal. Appl. 325, pp. 386-399 (2007). 
[89] Luckhardt, H., Extensional Gödel Functional Interpretation. Springer Lecture Notes in Mathematics 306, 1973.

[90] Macintyre, A., The mathematical significance of proof theory. Phil. Trans. R. Soc. A 363, pp. 2419-2435 (2005).

[91] Maehara, S., Eine Darstellung der intuitionistischen Logik in der Klassischen. Nagoya mathematical journal 7, pp. 45-64 (1954).

[92] McKinsey, J.C.C., Tarski, A., Some theorems about the sentential calculi of Lewis and Heyting. J. Symb. Logic 13, pp. 1-15 (1948).

[93] Oliva, P., Unifying functional interpretations. Notre Dame Journal of Formal Logic 47, pp. 263-290 (2006).

[94] Oliva, P., Understanding and using Spector's bar recursive interpretation of classical analysis. In: Proceedings of CiE 2006, Springer LNCS 3988, pp. 423-434 (2006).

[95] Parsons, C., On n-quantifier induction. J. Symbolic Logic 37, pp. 466-482 (1972).

[96] Rasiowa, H., Sikorski, R., Algebraic treatment of the notion of satisfiability. Fund. Math. 40, pp. 62-95 (1953).

[97] Schwichtenberg, H., Proof theory: Some aspects of cut-elimination. In: Barwise, J. (ed.) The Handbook of Mathematical Logic, pp. 867-895, North-Holland, Amsterdam 1977.

[98] Schwichtenberg, H., On bar recursion of types 0 and 1. J. Symbolic Journal 44, pp. 325-329 (1979).

[99] Shoenfield, J.S., Mathematical Logic. Addison-Wesley Publishing Company (Reading, Massachusetts) 1967.

[100] Simpson, S.G., Subsystems of Second Order Arithmetic. Perspectives in Mathematical Logic. Springer-Verlag. xiv+445 pp. 1999.

[101] Specker, E., Nicht konstruktiv beweisbare Sätze der Analysis. J. Symb. Logic 14, pp. 145-158 (1949).

[102] Spector, C., Provably recursive functionals of analysis: a consistency proof of analysis by an extension of principles formulated in current intuitionistic mathematics. In: Recursive function theory, Proceedings of Symposia in Pure Mathematics, vol. 5 (J.C.E. Dekker (ed.)), AMS, Providence, R.I., pp. 1-27 (1962).

[103] Stein, M., Interpretation der Heyting-Arithmetik endlicher Typen. Archiv für mathematische Logik und Grundlagenforschung 19, pp. 175-189 (1978).

[104] Streicher, T., Kohlenbach, U., Shoenfield is Gödel after Krivine. Math. Log. Quart. 53, pp. 176-179 (2007).

[105] Streicher, T., Reus, B., Classical logic: Continuation Semantics and Abstract Machines. Journal of Functional Programming 8(6), pp. 543-572 (1998).

[106] Tao, T., Soft analysis, hard analysis, and the finite convergence principle. Essay posted May 23, 2007. Available at: http://terrytao.wordpress.com/2007/05/23/soft-analysis-hard-analysisand-the-finite-convergence-principle/.

[107] Tao, T., Norm convergence of multiple ergodic averages for commuting transformations. arXiv:0707.1117v1 [math.DS] (2007). 
[108] Troelstra, A.S. (ed.) Metamathematical investigation of intuitionistic arithmetic and analysis. Springer Lecture Notes in Mathematics 344 (1973).

[109] Troelstra, A.S., Realizability. In: [16], pp. 407-473 (1998).

[110] Wang, H., Reflections on Kurt Gödel. 2nd printing. MIT Press (1988).

[111] Yasugi, M., Intuitionistic analysis and Gödel's interpretation. J. Math. Soc. Japan 15, pp. 101-112 (1963). 